\title{
INVESTIGATING PLANET FORMATION IN CIRCUMSTELLAR DISKS: CARMA OBSERVATIONS OF RY Tau AND DG Tau
}

\author{
Andrea Isella, John M. Carpenter, and Anneila I. Sargent \\ Division of Physics, Mathematics and Astronomy, California Institute of Technology, MC 249-17, Pasadena, CA 91125, USA; isella@ astro.caltech.edu \\ Received 2009 August 12; accepted 2010 March 22; published 2010 April 22
}

\begin{abstract}
We present CARMA observations of the thermal dust emission from the circumstellar disks around the young stars RY Tau and DG Tau at wavelengths of $1.3 \mathrm{~mm}$ and $2.8 \mathrm{~mm}$. The angular resolution of the maps is as high as 0 .' 15 , or $20 \mathrm{AU}$ at the distance of the Taurus cloud, which is a factor of 2 higher than has been achieved to date at these wavelengths. The unprecedented detail of the resulting disk images enables us to address three important questions related to the formation of planets. (1) What is the radial distribution of the circumstellar dust? (2) Does the dust emission show any indication of gaps that might signify the presence of (proto-)planets? (3) Do the dust properties depend on the orbital radius? We find that modeling the disk surface density in terms of either a classical power law or the similarity solution for viscous disk evolution reproduces the observations well. Both models constrain the surface density between 15 and 50 AU to within $30 \%$ for a given dust opacity. Outside this range, the densities inferred from the two models differ by almost an order of magnitude. The $1.3 \mathrm{~mm}$ image from RY Tau shows two peaks separated by 0 '.2 with a decline in the dust emission toward the stellar position, which is significant at about $2 \sigma-4 \sigma$. For both RY Tau and DG Tau, the dust emission at radii larger than 15 AU displays no significant deviation from an unperturbed viscous disk model. In particular, no radial gaps in the dust distribution are detected. Under reasonable assumptions, we exclude the presence of planets more massive than 5 $M_{J}$ orbiting either star at distances between about 10 and $60 \mathrm{AU}$, unless such a planet is so young that there has been insufficient time to open a gap in the disk surface density. The radial variation of the dust opacity slope, $\beta$, was investigated by comparing the $1.3 \mathrm{~mm}$ and $2.8 \mathrm{~mm}$ observations. We find mean values of $\beta$ of 0.5 and 0.7 for DG Tau and RY Tau, respectively. Variations in $\beta$ are smaller than $\Delta \beta=0.7$ between 20 and 70 AU. These results confirm that the circumstellar dust throughout these disks differs significantly from dust in the interstellar medium.
\end{abstract}

Key words: accretion, accretion disks - stars: pre-main sequence - stars: variables: T Tauri, Herbig Ae/Be submillimeter: planetary systems - techniques: interferometric

Online-only material: color figures

\section{INTRODUCTION}

Resolved images of circumstellar disks around young stars provide the most direct tool for investigating the formation of planets. At millimeter wavelengths, the thermal dust emission is generally optically thin and measures the radial distribution of circumstellar dust (Beckwith et al. 1990). However, since circumstellar disks in nearby star-forming regions typically have radii between 100 and $500 \mathrm{AU}$, sub-arcsecond angular resolution is required to spatially resolve the dust emission, even in nearby star-forming clouds. Millimeter-wave interferometers are essential for such studies.

Since sub-arcsecond observations at millimeter wavelengths require both high sensitivity and high dynamical range, only a small number of bright disks have been observed at resolutions of 0.4-1" to date (Brown et al. 2008; Guilloteau et al. 1999; Isella et al. 2007; Piétu et al. 2005, 2006, 2007; Simon et al. 2000; Testi et al. 2003; Wilner et al. 2000). The Combined Array for Research in Millimeter-wave Astronomy (CARMA) and the new extended configuration of the Sub-millimeter Array are rapidly enabling more extensive high-resolution surveys of circumstellar disks, particularly in the Taurus and Ophiuchus star-forming regions (Andrews et al. 2009; Hughes et al. 2009; Isella et al. 2009, hereafter Paper I).

The highest angular resolution achieved so far by millimeterwave interferometers is $0.3-00^{\prime \prime} 4$, corresponding to spatial scales of 40-50 AU at the distance of Taurus and Ophiuchus. In most cases, the dust density appears to increase smoothly inward down to the orbital radius resolved by the observations, typically $\sim 25$ AU. However, central cavities in the dust distribution are revealed in a number of disks (Andrews et al. 2009; Hughes et al. 2009). It remains a matter of debate whether these cavities are caused by dynamical interactions, inside-out disk dispersal mechanisms, dust opacity variations, or viscous evolution (e.g., Alexander et al. 2006; Calvet et al. 2005; Chiang \& Murray-Clay 2007; Dullemond \& Dominik 2005; Paper I).

Nevertheless, these observations still lack the angular resolution required to resolve the innermost part of the disk where the density of the circumstellar material is highest and the formation of planets is more probable. Here we describe CARMA observations of the thermal dust emission toward the young stars DG Tau and RY Tau at an angular resolution of 0 ". 15 at $1.3 \mathrm{~mm}$ and 0.3 at $2.8 \mathrm{~mm}$. At the distance of Taurus $(140 \mathrm{pc})$, 0 .' 15 corresponds to spatial scales of $20 \mathrm{AU}$, such that emission on orbital scales comparable to Saturn can be resolved. This is more than a factor of 2 improvement over previous observations of circumstellar disks at these wavelengths.

DG Tau and RY Tau are classical T Tauri stars of spectral type M0 and K1, respectively (Muzerolle et al. 1998; Kenyon \& Hartmann 1995). Stellar ages inferred from stellar evolutionary models are less than 1 Myr (see Paper I for more details and references). The relative youth of both systems is confirmed by the presence of large amounts of gas and dust extending to $0.1 \mathrm{pc}$ and by associated stellar jets and outflows (see, e.g., McGroarty \& Ray 2004; St-Onge \& Bastien 2008). From near-infrared to millimeter wavelengths, both objects exhibit strong emission in 
Table 1

Summary of CARMA Continuum Observations

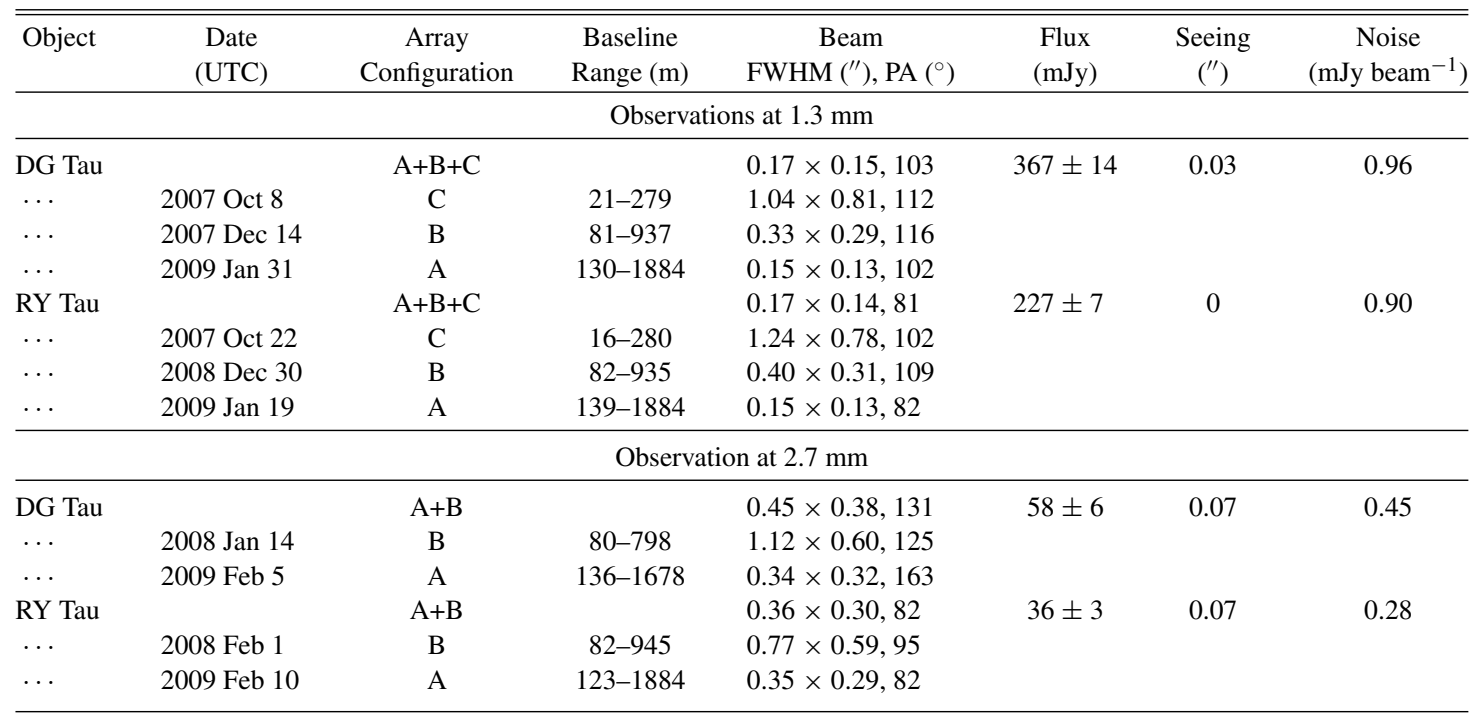

excess of that from the stellar photospheres. This is attributed to rotating disks with radii of few hundred AU that first absorb and then re-emit radiation from the central stars (Koerner \& Sargent 1995; Testi et al. 2002). Our earlier CARMA observations of $1.3 \mathrm{~mm}$ thermal dust emission from these disks, at a resolution of 0.7 , suggested disk masses between $5 \%$ and $150 \%$ of the stellar mass for both sources (see Paper I). These high disk masses and the youth of RY Tau and DG Tau make these prime targets to investigate the earliest stages of planet formation. Our new observations of RY Tau and DG Tau have a factor of 5 better angular resolution and a factor of 3 better sensitivity than the previous data.

This paper investigates three main questions related to the formation of planets in young circumstellar disks. (1) What is the surface density distribution in the observed disks down to an orbital radius of $10 \mathrm{AU}$ ? (2) Are there any signatures of planet formation contained in the dust distribution? Finally, (3), do the dust properties vary with orbital radius? A qualitative answer to the first two questions is proposed in Section 3 where we present the observations and discuss the morphology of the dust emission. A quantitative analysis is described in Section 4, where we compare the observations with theoretical models of disk emission. Implications of these results for disk structure, for the possible presence of planets, and for the radial variation of the dust opacity are considered in Section 5. The conclusions are presented in Section 6.

\section{OBSERVATIONS AND DATA REDUCTION}

We observed thermal dust emission from the RY Tau and DG Tau circumstellar disks using CARMA in the A, B, and $\mathrm{C}$ configurations. The date of observation, array configurations used, baseline range, sizes and orientations of the synthesized beams, integrated fluxes, seeing, and noise levels are summarized in Table 1. The C-configuration observations were presented in Paper I.

The observations were obtained at local oscillator frequencies of $228.1 \mathrm{GHz}(\lambda=1.3 \mathrm{~mm})$ and $106.2 \mathrm{GHz}(\lambda=2.8 \mathrm{~mm})$. The CARMA correlator at the time of the observations contained three bands, each of which was configured to $468 \mathrm{MHz}$ bandwidth to provide maximum continuum sensitivity. The bandpass shape was calibrated by observing $3 \mathrm{C} 273$; flux calibration was set by observing Uranus and $3 \mathrm{C} 84$. The radio galaxy $3 \mathrm{C} 111$ was observed every 9 minutes to correct for atmospheric and instrumental effects. Variations of the atmospheric conditions on timescales shorter than 9 minutes are not corrected, in effect resulting in seeing. We quantified the atmospheric seeing by measuring the size of the phase calibrator image; if the seeing is negligible, the phase calibrator appears as a point source. Otherwise, the seeing produces a Gaussian smoothing that can be quantified through the full width at half-maximum (FWHM) of the resulting image. We find that at $1.3 \mathrm{~mm}$ the effect of seeing is negligible for RY Tau but produces an FWHM of 0 '.03 for DG Tau. Atmospheric conditions were slightly worse during the $2.8 \mathrm{~mm}$ observations, resulting in seeing of 0.07 for both objects. These seeing estimates do not account for variations in the atmospheric conditions on angular scales of $10^{\circ}$, corresponding to the separation between the source and the calibrator. Values for the atmospheric seeing are summarized in Table 1 and are adopted in the model fitting described in Section 4.

The raw data were reduced using the MIRIAD software package. The maps of the continuum emission shown in Figure 1 were derived using GILDAS software. Corresponding complex visibilities are shown in Figure 2. At $1.3 \mathrm{~mm}$, natural weighting of the $\mathrm{A}, \mathrm{B}$, and $\mathrm{C}$ configuration observations produced an FWHM synthesized beam size of $\sim 0$ ! 15 . The noise levels are $0.96 \mathrm{mJy} \mathrm{beam}^{-1}$ and $0.90 \mathrm{mJy}^{\text {beam }}{ }^{-1}$, respectively, for DG Tau and RY Tau. Dust emission at $2.8 \mathrm{~mm}$ was observed in the $\mathrm{A}$ and $\mathrm{B}$ configurations at angular resolution of $\sim 0^{\prime \prime} .35$ and noise levels of $0.45 \mathrm{mJy}^{\text {beam }}{ }^{-1}$ and $0.28 \mathrm{mJy} \mathrm{beam}^{-1}$ for DG Tau and RY Tau, respectively.

\section{MORPHOLOGY OF THE DUST EMISSION}

In Figure 1, the dust emission in both disks is clearly resolved and characterized by a smooth and centrally symmetric radial profile. DG Tau intensity contours are almost circular suggesting a disk inclination smaller than $30^{\circ}$. For RY Tau, the intensity contours are elongated in the northeast direction suggesting a disk position angle of about $24^{\circ}$ measured east from north and a disk inclination of at least $65^{\circ}$. For both sources, the disk orientations agree with those found in Paper I. 

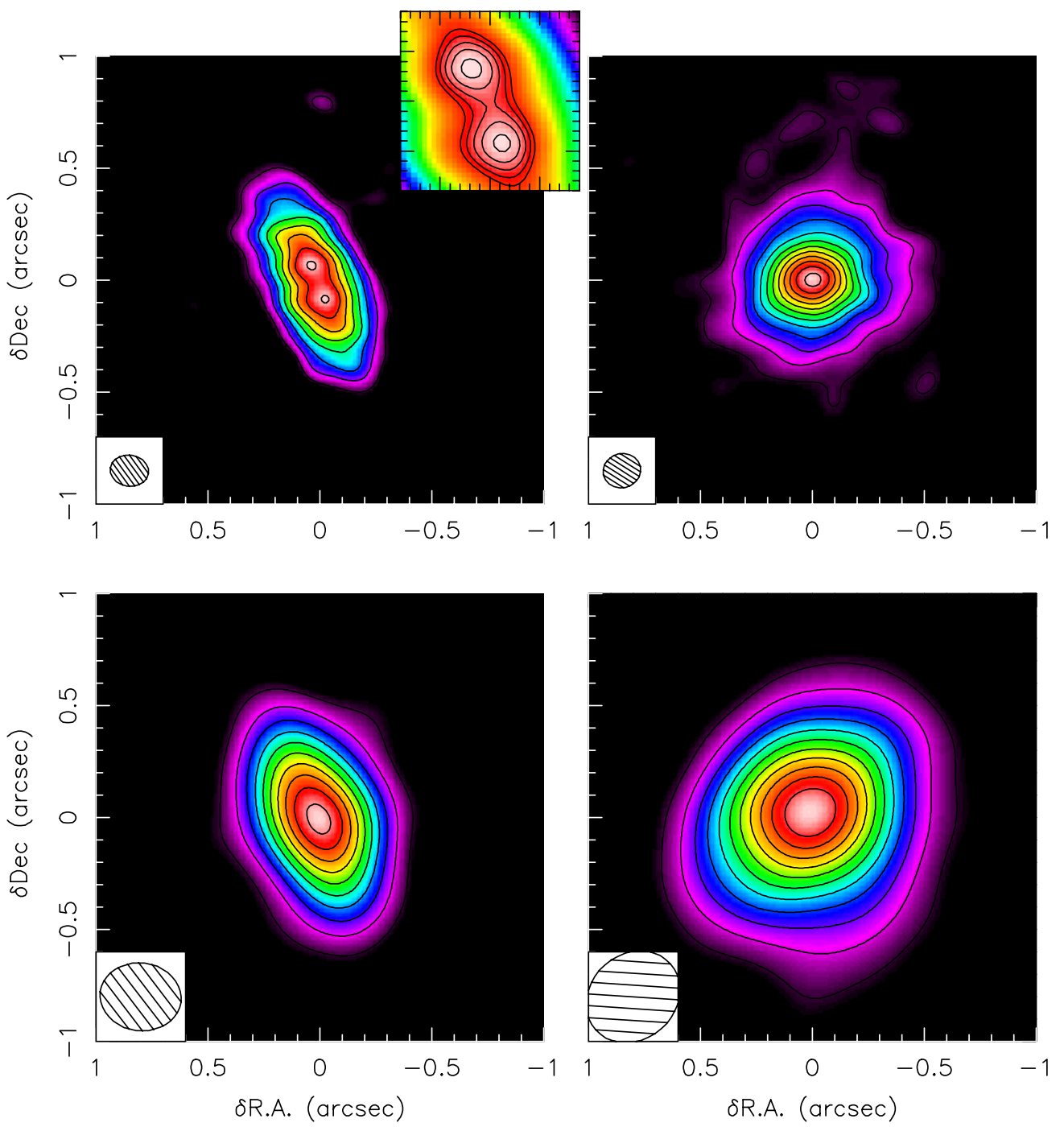

Figure 1. Maps of the dust thermal emission observed at a wavelength of $1.3 \mathrm{~mm}$ (upper panels) and $2.8 \mathrm{~mm}$ (lower panels) toward RY Tau (left panels) and DG Tau (right panels). The color scale shows the surface brightness starting from the $3 \sigma$ level, with contours plotted every $4 \sigma$. The $1 \sigma$ noise level and the size of the synthesized beam are given in Table 1 . The inset in the upper left panel shows the central $0^{\prime \prime} 4 \times 00^{\prime \prime} 4$ region of the RY Tau disk where contours start at $28 \sigma$ with increments of $1 \sigma$. The surface brightness is characterized by two peaks separated by $\sim 0^{\prime \prime} 2$

(A color version of this figure is available in the online journal.)

\subsection{RY Tau Disk Morphology}

The $1.3 \mathrm{~mm}$ dust continuum emission from the RY Tau disk shows two spatially resolved peaks separated by about 0.2 (28 AU), and oriented along the apparent major axis of the disk. Details of the central $0{ }^{\prime} .4 \times 00^{\prime \prime} 4$ region are displayed in the inset in the upper left panel of Figure 1, and the radial profile of the surface density along the disk major axis is shown in Figure 3 . The intensity at both peaks is $29 \mathrm{mJy}$ beam $^{-1}$, which is $2 \mathrm{mJy}$ beam $^{-1}$ (i.e., $2.2 \sigma$ ) higher than the intensity at the center of the disk. We also estimated the expected central surface brightness by fitting a Gaussian to the surface brightness distribution at angular distances larger than 0.15 . The fitted Gaussian is shown as the solid curve in Figure 3. A Gaussian function was chosen since it provides a reasonable parametric representation of the dust emission. Interpolating this Gaussian fit to the center of the disk suggests an expected central surface brightness of $31 \mathrm{mJy}^{\text {beam }}{ }^{-1}$, which is $4 \sigma$ higher than the measured value. The significance level of the two intensity peaks, the fact that they appear in the map before cleaning, their orientation along the disk major axis, and the symmetry with respect to the central star, suggests that they are real and, therefore, that the dust emission decreases inside an orbital radius of about $14 \mathrm{AU}$. This is analogous to the situation in "transitional" disks, where the inner gaps observed in the dust emission are attributed to dusty depleted inner regions (see, e.g., Hughes et al. 2009; Brown et al. 2008, 2009).

At a first sight, this interpretation is incompatible with RY Tau's large near- and mid-infrared excesses, which suggest the presence of warm dust within $10 \mathrm{AU}$ of the star (Robitaille et al. 2007). If, however, the inner disk is only partially depleted and dust emission remains optically thick in the infrared, the observed double intensity peak and the spectral energy distribution (SED) can be reconciled. A number of physical mechanisms could reduce the dust density in the inner region of circumstellar disks. For example, planets less massive than Jupiter may carve partially depleted gaps in the surface density distribution by tidal interaction with the surrounding material (Bryden et al. 1999). This possibility is discussed in more detail in Section 5.3. In Paper I, we also proposed that a surface density profile that gradually decreases toward the star may originate naturally from the viscous evolution of a disk if viscosity 

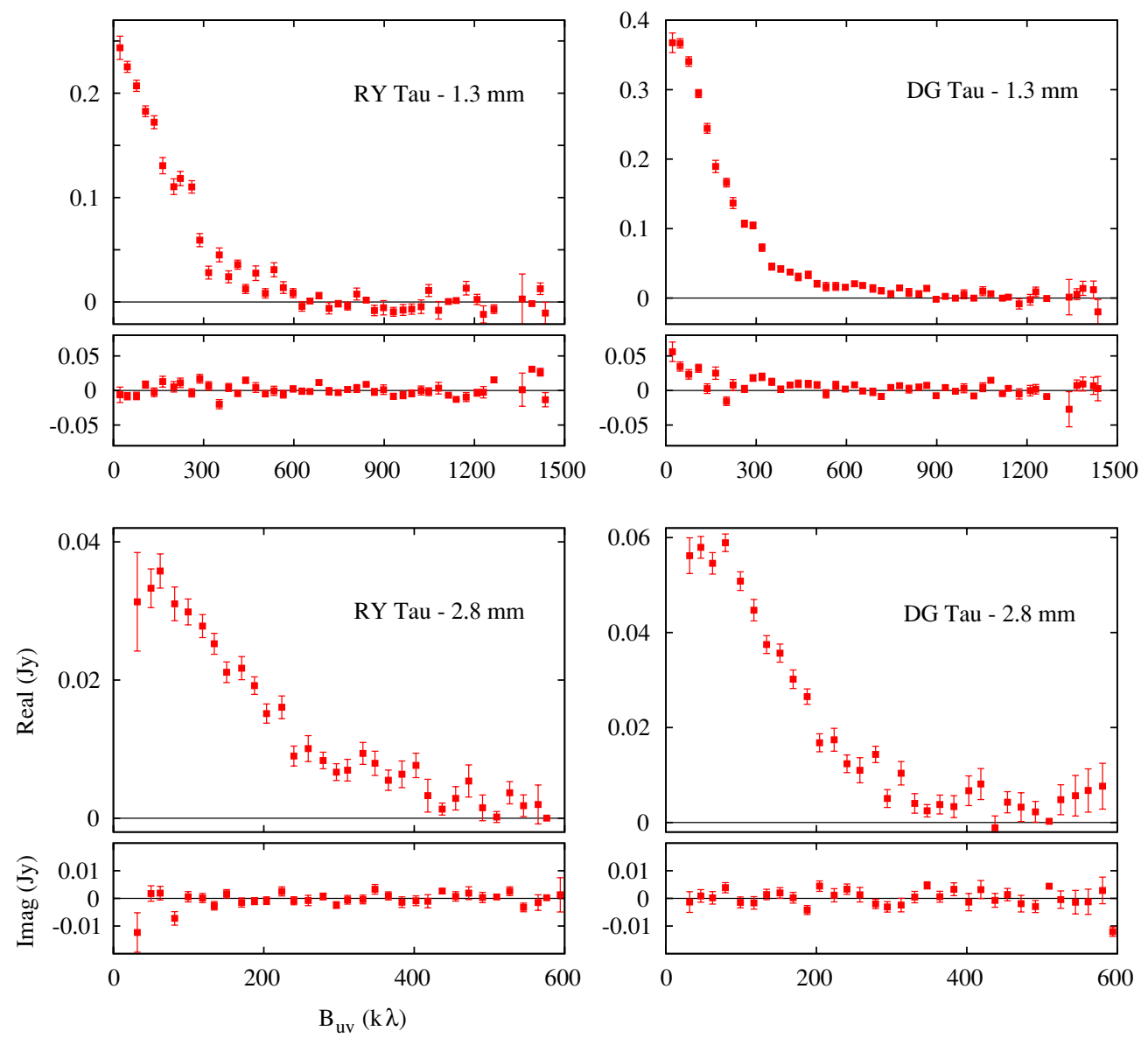

Figure 2. Correlated flux measured at $1.3 \mathrm{~mm}$ and $2.8 \mathrm{~mm}$ toward RY Tau (left panels) and DG Tau (right panels) as a function of the baseline length $B_{u v}$ expressed in $\mathrm{k} \lambda$.

(A color version of this figure is available in the online journal.)

decreases with radius. Finally, it is also possible that the decrease in dust emission may originate from a lowering of opacity due to the growth of dust grains to centimeter sizes (Dullemond \& Dominik 2005). Unambiguously disentangling these models requires even higher angular resolution observations than are yet available.

We must note that radial velocity studies (Herbig \& Bell 1988) and Hipparcos observations of the variability of the photocenter (Bertout et al. 1999) suggest that RY Tau is a binary. The Hipparcos data imply a minimum projected separation of 23.6 mas and a position angle of $304^{\circ} \pm 34^{\circ}$, almost perpendicular to the position angle of the disk inferred from our observations. Assuming that the binary and the disk have the same inclination, the spatial separation between the binary components is 6-9 AU, and could explain the double peak in the dust continuum emission. Indeed, the presence of a stellar mass companion orbiting at a radius of 6-9 AU would push the inner radius of the circumstellar disk to a distance of 9-13 AU by tidal interactions (Wolf et al. 2007). However, the binary nature of RY Tau has been rendered questionable by near-infrared interferometric observations that suggest an inner disk radius at $0.1 \mathrm{AU}$ from the central star and exclude the presence of a stellar mass companion between $0.35 \mathrm{AU}$ and $4 \mathrm{AU}$ down to a stellar flux ratio of 0.05 (Akeson et al. 2005; Pott et al. 2010). A stellar companion was also undetected in recent spectroscopic and aperture masking observations (D. C. Nguyen \& A. Kraus 2009, private communication). As discussed above, the SED is also inconsistent with the existence of a large inner gap completely depleted of gas and dust as could be expected for a stellar companion (Robitaille et al. 2007). These results suggest that RY Tau is indeed a single star, and the variability observed by Hipparcos and the radial velocity variations may be attributed to brightness changes in the circumstellar environment (see the discussion in Schegerer et al. 2008, and references therein).

A notable characteristic of our images of the dust emission is the high degree of central symmetry and, with the exception of the innermost region, the almost complete absence of features in the surface brightness distribution. If the emission is optically thin (we will examine this assumption is Section 5.3), this translates to a smooth radial profile for the dust. The degree of symmetry of the emission can be quantified by analyzing the imaginary part of the correlated flux, plotted in Figure 2 as a function of the angular frequency $B_{u v}$. Point-symmetric emission will have a zero imaginary part at all spatial frequencies. For RY Tau, the deviations from zero are comparable to the noise in the observations (see the left panels of Figure 2).

\subsection{DG Tau Disk Morphology}

The surface brightness distribution for the DG Tau disk has a central peak coincident with the stellar position and smoothly decreases outward to reach the noise level at an angular distance 


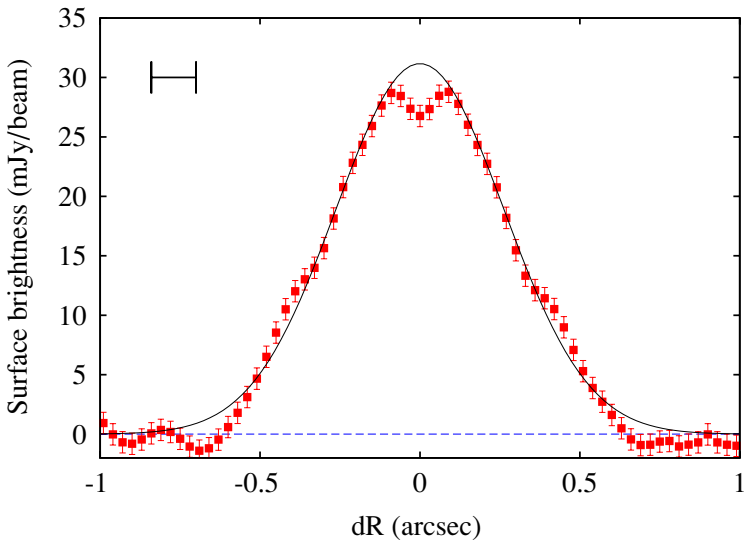

Figure 3. Radial profile of the $1.3 \mathrm{~mm}$ surface brightness in the RY Tau disk measured along the major axis of the disk at a position angle of $24^{\circ}$ east from north. The intensity error bars (red points) correspond to the noise level of $0.9 \mathrm{mJy}$ beam $^{-1}$. The solid curve shows a Gaussian fit, while the dashed line indicates the zero intensity level. The bracket in the upper left indicates the angular resolution.

(A color version of this figure is available in the online journal.)

of about 0 '.5. At both 1.3 and $2.8 \mathrm{~mm}$ the emission appears fairly symmetric, and indeed the imaginary part of the correlated flux is zero for $B_{u v}>300 \mathrm{k} \lambda$ (see the upper right panel of Figure 2). The imaginary part increases up to $50 \mathrm{mJy}$ at shorter spatial frequencies, suggesting that the emission may be asymmetric on angular scales larger than $1 / B_{u v} \sim 0^{\prime \prime}$.7. Comparing the real and imaginary parts of the correlated flux at the shortest spatial frequencies, we find that the asymmetric part of the emission contributes about $14 \%$ to the total flux.

As already noted, the high angular resolution observations of RY Tau and DG Tau allow us to examine disk properties that bear on planet formation. In particular, the radial density profile of the circumstellar material is critical to understanding where planet formation may occur, or where it has occurred. With observations at more than one wavelength, we may also consider radial variations of the grain properties. As we describe below, measurements of the dust radial profile and the variation of the dust properties with radius are best undertaken by comparing the observations with theoretical disk models in the Fourier domain. There, the effects of different angular resolutions, sensitivity, and atmospheric seeing can be more easily taken into account. Moreover, comparison with theoretical models is the only way to quantify the contribution from optically thick emission, leading to an improved estimate of the dust mass.

\section{DISK AND DUST MODELS}

To investigate the dust radial distribution around DG Tau and RY Tau, we consider two different models for the disk surface density. The first model consists of the classical powerlaw parameterization

$$
\Sigma(R)=\Sigma_{40}\left(\frac{R}{40 \mathrm{AU}}\right)^{-p} \text { for } R_{\mathrm{in}}<R<R_{\mathrm{out}},
$$

where $\Sigma_{40}$ is the disk surface density at a radius of 40 AU. $R_{\text {in }}$ and $R_{\text {out }}$ are the inner and outer disk radii, respectively. The second model is the similarity solution for the evolution of a viscous Keplerian disk (Lynden-Bell \& Pringle 1974; Hartmann et al. 1998). As discussed in Paper I, this has the form
$\Sigma(R, t)=\Sigma_{t}\left(\frac{R_{t}}{R}\right)^{\gamma} \times \exp \left\{-\frac{1}{2(2-\gamma)}\left[\left(\frac{R}{R_{t}}\right)^{(2-\gamma)}-1\right]\right\}$,

where $\Sigma_{t}$ is the surface density at radius $R_{t}$, sometimes called the transition radius. For $R \lesssim R_{t}$, the surface density has a power-law profile characterized by the slope $\gamma$, while at larger radii the surface density falls exponentially.

These two different parameterizations are used to calculate the dust emission by solving the structure of an hydrostatic disk heated by the stellar radiation (Dullemond et al. 2001). The temperature on the disk mid-plane is self-consistently calculated by adopting the two-layer approximation of Chiang \& Goldreich (1997). The disk temperature, which depends mainly on the disk optical depth at optical and infrared wavelengths, is roughly proportional to $R^{-1 / 2}$ for both surface density profiles (see Paper I and references therein for a detailed discussion on the disk temperature radial profile).

Fundamental to any disk model is the prescription adopted for the dust opacity. Although the dust contributes only about $1 \%$ to the total disk mass, it dominates the disk opacity in the wavelength range where most of the stellar and disk radiation is emitted. We assume that the dust size distribution follows a power law $n(a) \propto a^{-q}$, where $a$ is the radius of a dust grain. The assumptions on the slope $q$, on the minimum and maximum grain sizes, on the dust chemical composition and on the grain structure define the frequency dependence of the dust opacity $k_{v}$, and, ultimately, the disk emission. The dust opacity is calculated for compact spheres composed of astronomical silicates and organic carbonates (Weingartner \& Draine 2001; Zubko et al. 1996). We assume a mass ratio of 1 between silicates and organics, which leads to grain density of $2.5 \mathrm{~g} \mathrm{~cm}^{-3}$. The dust opacity averaged over the grain size distribution is calculated by fixing the minimum grain size to $0.005 \mu \mathrm{m}$. The maximum grain size $a_{\max }$ and the slope $q$ are set to reproduce the observed slope of the SED as discussed below.

At millimeter wavelengths, the dust opacity can be approximated by a power law $k_{v}=k_{0}\left(v / v_{0}\right)^{\beta}$ (Beckwith \& Sargent 1991). If the dust emission is optically thin and the Rayleigh-Jeans approximation is satisfied, the slope $\beta$ of the dust opacity is related to the spectral index $\alpha$ of the observed disk emission $F_{v}\left(F_{v} \propto v^{\alpha}\right)$ by the relation $\alpha=2+\beta$. This relation is only approximate if the dust emission is optically thick at some radii. In Paper I, we derived values for $\beta$ of 0.5 and 0.7 for DG Tau and RY Tau, respectively, from an analysis of the SED, taking into account the optically thick contribution to the total dust emission. For the assumed dust composition and structure, these values of $\beta$ can be reproduced with different choices of the maximum grain size $a_{\max }$ and the grain size slope $q$ (see the Appendix). To investigate how the assumptions on the grain size distribution affect the model fitting, we adopt two different dust models that correspond to the extreme cases of low $(L)$ and high $(H)$ opacity. The corresponding dust opacities at both $1.3 \mathrm{~mm}$ and $2.8 \mathrm{~mm}$ are given in Table 2 .

Finally, we assume that the dust opacity is constant throughout the disk. This is indeed one of the main assumptions we want to test by modeling the observed dust emission at $1.3 \mathrm{~mm}$ and $2.8 \mathrm{~mm}$ and will be discussed in detail in Section 5.4.

\section{RESULTS AND DISCUSSION}

Models and observations are compared in Fourier space to avoid the nonlinear effects introduced by the cleaning process. The best-fit models are found by $\chi^{2}$ minimization with five free 
Table 2

Properties of the Adopted Dust Models

\begin{tabular}{lcccc}
\hline \hline Object & $\begin{array}{c}a_{\max } \\
(\mathrm{cm})\end{array}$ & $q$ & $\begin{array}{c}k_{1.3 \mathrm{~mm}} \\
\left(\mathrm{~cm}^{2} \mathrm{~g}^{-1}\right)\end{array}$ & $\begin{array}{c}k_{2.8 \mathrm{~mm}} \\
\left(\mathrm{~cm}^{2} \mathrm{~g}^{-1}\right)\end{array}$ \\
\hline DG Tau & \multicolumn{5}{c}{ High dust opacity model, $H$} \\
RY Tau & 0.075 & 3 & 0.082 & 0.056 \\
\hline & 3 & 0.131 & 0.075 \\
\hline DG Tau & 5.0 & Low dust opacity model, $L$ & 0.0078 \\
RY Tau & 5.0 & 3.5 & 0.012 & 0.015 \\
\hline
\end{tabular}

parameters: the disk inclination $i$, the disk position angle PA, $R_{\text {out }}, \Sigma_{40}$, and $p$ for the power-law surface density (Equation (1)), and $i, \mathrm{PA}, R_{t}, \Sigma_{t}$, and $\gamma$ for the similarity solution (Equation (2)). The disk inner radius $R_{\text {in }}$ is fixed at 0.1 AU. For both surface density models, we find best-fit solutions for both the high $(H)$ and low $(L)$ dust opacity models. The $1.3 \mathrm{~mm}$ and $2.8 \mathrm{~mm}$ data are fitted independently.

To minimize $\chi^{2}$ and evaluate the constraints on the model parameters, we use a Bayesian approach that adopts uniform prior probability distributions. In practice, we sample the $\chi^{2}$ probability distribution by varying the free parameters using the Markov Chain Monte Carlo method described in Paper I.

Once a best-fit solution is found, we confirm that this indeed corresponds to an absolute minimum of $\chi^{2}$, as opposed to a local minimum, by running multiple Monte Carlo simulations with random initializations and verifying that they all converge to the same solution. Each parameter is allowed to vary in a large range: $0^{\circ}-80^{\circ}$ for the inclination, $\pm 90^{\circ}$ for the position angle, 10-1000 AU for $R_{t}$ and $R_{\text {out }}, \pm 4$ for $p$ and $\gamma$, and 0.1$1000 \mathrm{~g} \mathrm{~cm}^{-2}$ for $\Sigma_{40}$ and $\Sigma_{t}$.

The best-fit disk models found for high and low dust opacities are listed in Tables 3 and 4, respectively. Each table lists the parameters for the similarity solution disk model in the upper part, and for the power-law disk model in the lower part. The probability distributions for each free parameter are shown in Figures 4 and 5 for RY Tau in the case of the similarity solution and power law, respectively. The same quantities for DG Tau are shown in Figures 6 and 7. In these figures, the black and red histograms indicate the probability distributions derived by fitting the $1.3 \mathrm{~mm}$ and $2.8 \mathrm{~mm}$ observations, respectively; solid and dashed curves represent the $H$ and $L$ dust opacity models. For each parameter, we derive the uncertainty range that corresponds to a likelihood of $99.7 \%(3 \sigma)$ by fitting a normal distribution to the probabilities.

Finally, Figure 8 shows comparisons between the observed real part of the correlated flux (filled squares with error bars), the best-fit models for the similarity solution (solid curve), and a power-law surface density (dashed curve).

\subsection{Dependence on the Dust Opacity and Implications on the Disk Masses}

The best-fit solutions for the $H$ and $L$ dust opacity models are shown in Figures 5 and 6 with solid and dashed curves, respectively. In all cases, $H$ and $L$ models lead to very similar values for the disk position angle, the disk inclination and the radial profiles of the surface density defined by $p$ and $R_{\text {out }}$ in the case of the power-law models, and $\gamma$ and $R_{t}$ for the similarity solution models. As discussed in Paper I, these parameters are essentially independent of the dust opacity. This is mainly because the disk mid-plane temperature $T_{i}(R)$ varies by only a few percent between the different dust models, as long as the disk is optically thick to the stellar radiation. Since $\Sigma(R) \propto T_{i}(R)^{-1}$, the radial profile of the surface density varies by only small fraction when different dust models are assumed.

By contrast, the surface density normalization $\left(\Sigma_{t}\right.$ and $\left.\Sigma_{40}\right)$ varies with the dust opacity so that the product $\Sigma \times k_{v}$ remains almost constant if the emission is optically thin. Consequently, a lower dust opacity requires a higher dust mass in order to emit the same amount of radiation at millimeter wavelengths. The ratio $\Sigma_{L} / \Sigma_{H}$ is then approximately equal to the ratio between the dust opacities listed in Table 2.

From the analysis of the surface density of the best-fit model, we find that the RY Tau emission is always optically thin at both 1.3 and $2.8 \mathrm{~mm}$. However, DG Tau emission is optically thick within $20 \mathrm{AU}$ at $1.3 \mathrm{~mm}$ for both the similarity solution and the power-law models. The $1.3 \mathrm{~mm}$ flux emitted within this region is about $25 \%$ of the total flux. At $2.8 \mathrm{~mm}$ the emission is always optically thin in the case of the similarity solution, while it is optically thick within $6 \mathrm{AU}$ in the power-law case. In this case, the optically thick contribution is $5 \%$ of the total flux.

Different dust opacities lead to different values for the total mass of dust in the disks. For DG Tau, we obtain total dust masses of about 33 and 233 Earth masses $\left(M_{\oplus}\right)$ in the case of the high and low opacity dust models, respectively. Disk masses of $\sim 10$ and $50 M_{\oplus}$ are found for RY Tau. Massive disks can also be obtained by extending the grain size distribution larger than $5 \mathrm{~cm}$. For example, in Paper I we derived total dust

Table 3

Best-fit Parameters Assuming the $H$ Dust Model

\begin{tabular}{|c|c|c|c|c|c|c|c|}
\hline \multicolumn{8}{|c|}{ Similarity solution } \\
\hline Object & $\lambda(\mathrm{mm})$ & $i\left(^{\circ}\right)$ & $\mathrm{PA}\left({ }^{\circ}\right)$ & $R_{t}(\mathrm{AU})$ & $\gamma$ & $\Sigma_{t}\left(\mathrm{~g} \mathrm{~cm}^{-2}\right)$ & $\chi_{r}^{2}$ \\
\hline DG Tau & 1.3 & $24 \pm 9$ & $119 \pm 23$ & $23.4 \pm 1.8$ & $0.33 \pm 0.15$ & $10.9 \pm 1.5$ & 1.0608 \\
\hline RYTau & 1.3 & $66 \pm 2$ & $24 \pm 3$ & $26.7 \pm 1.2$ & $-0.54 \pm 0.18$ & $2.6 \pm 0.2$ & 1.0896 \\
\hline$\cdots$ & 2.8 & $71 \pm 6$ & $20 \pm 4$ & $26.5 \pm 2.7$ & $-0.08 \pm 0.54$ & $2.6 \pm 0.5$ & 1.1894 \\
\hline Object & $\lambda(\mathrm{mm})$ & $i\left(^{\circ}\right)$ & $\mathrm{PA}\left({ }^{\circ}\right)$ & $R_{\text {out }}(\mathrm{AU})$ & $p$ & $\Sigma_{40}\left(\mathrm{~g} \mathrm{~cm}^{-2}\right)$ & $\chi_{r}^{2}$ \\
\hline DG Tau & 1.3 & $27 \pm 8$ & $120 \pm 26$ & $72.6 \pm 6.3$ & $1.00 \pm 0.15$ & $5.6 \pm 1.5$ & 1.0611 \\
\hline$\ldots$ & 2.8 & $32 \pm 11$ & $144 \pm 18$ & $82.2 \pm 10.5$ & $0.74 \pm 0.24$ & $4.5 \pm 1.6$ & 1.0629 \\
\hline RYTau & 1.3 & $66 \pm 2$ & $24 \pm 3$ & $70.6 \pm 3.9$ & $0.12 \pm 0.15$ & $1.9 \pm 0.6$ & 1.0897 \\
\hline$\ldots$ & 2.8 & $71 \pm 6$ & $20 \pm 4$ & $76.9 \pm 12.0$ & $0.64 \pm 0.45$ & $1.6 \pm 1.0$ & 1.1894 \\
\hline
\end{tabular}

Note. The uncertainties correspond to a likelihood of $99.7 \%$ (i.e., $3 \sigma$ ) for the normal distributions shown in Figures 5 and 6. 

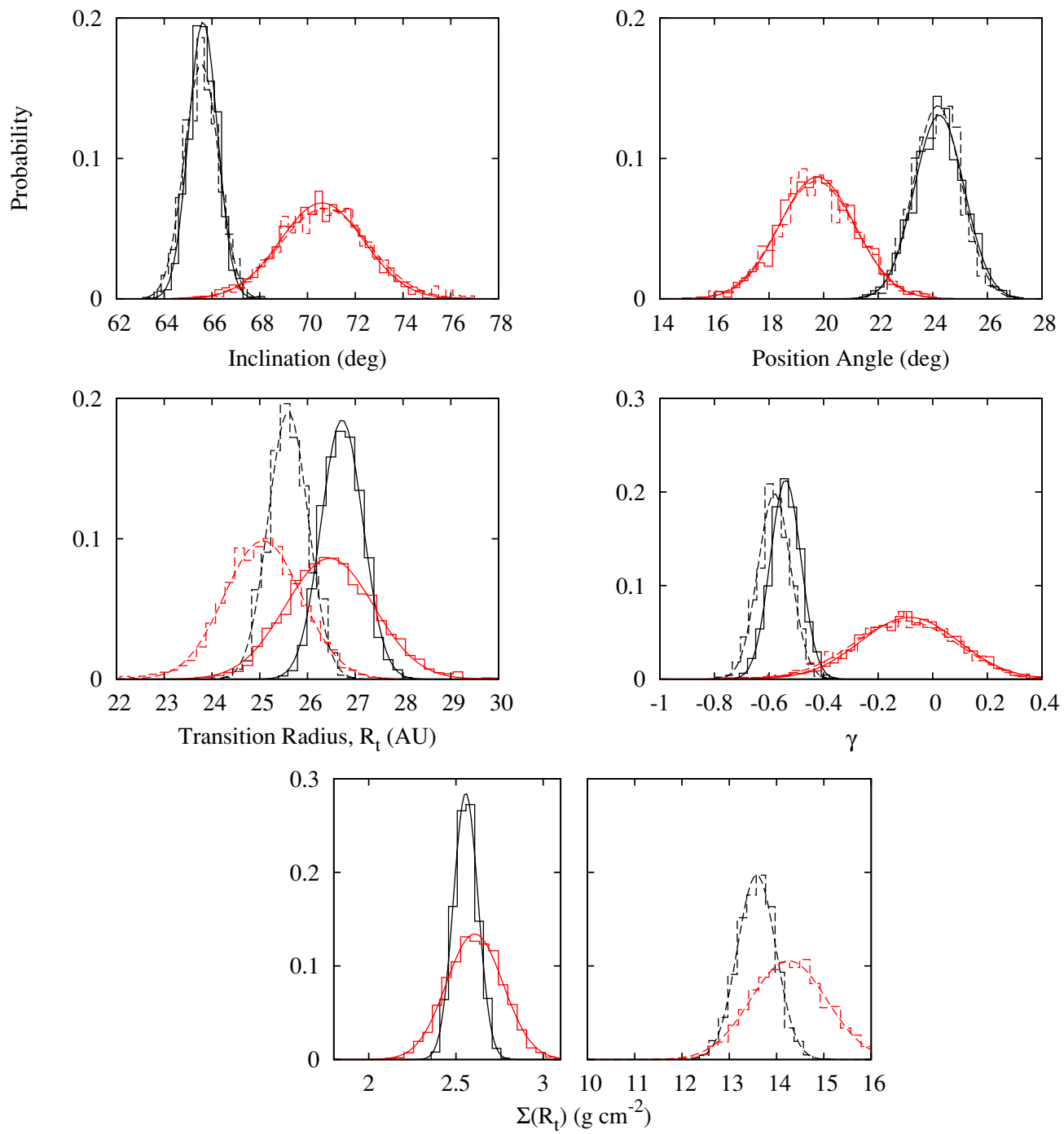

Figure 4. Probability distribution of the disk parameters obtained by fitting the RY Tau observations at $1.3 \mathrm{~mm}$ and $2.8 \mathrm{~mm}$ with the similarity solution for the surface density distribution. The results obtained by fitting the $1.3 \mathrm{~mm}$ data only are shown by the black curves, while the red curves indicate the results obtained by fitting the $2.8 \mathrm{~mm}$ only. Solid curves show the probability distribution obtained assuming the dust opacity model $H$, and dashed curves correspond to the dust opacity model $L$ (see Table 2).

(A color version of this figure is available in the online journal.)

Table 4

Best-fit Parameters Assuming the $L$ Dust Model

\begin{tabular}{|c|c|c|c|c|c|c|c|}
\hline \multicolumn{8}{|c|}{ Similarity solution } \\
\hline Object & $\lambda(\mathrm{mm})$ & $i\left(^{\circ}\right)$ & $\mathrm{PA}\left({ }^{\circ}\right)$ & $R_{t}(\mathrm{AU})$ & $\gamma$ & $\Sigma_{t}\left(\mathrm{~g} \mathrm{~cm}^{-2}\right)$ & $\chi_{r}^{2}$ \\
\hline DG Tau & 1.3 & $24 \pm 11$ & $119 \pm 24$ & $22.5 \pm 1.8$ & $0.28 \pm 0.15$ & $74.4 \pm 9.9$ & 1.0608 \\
\hline$\ldots$ & 2.8 & $31 \pm 12$ & $144 \pm 20$ & $26.4 \pm 2.7$ & $0.07 \pm 0.27$ & $55.4 \pm 8.9$ & 1.0629 \\
\hline RYTau & 1.3 & $66 \pm 2$ & $24 \pm 3$ & $25.6 \pm 1.2$ & $-0.58 \pm 0.18$ & $13.6 \pm 1.2$ & 1.0896 \\
\hline$\ldots$ & 2.8 & $71 \pm 6$ & $20 \pm 4$ & $25.1 \pm 2.4$ & $-0.10 \pm 0.57$ & $14.3 \pm 2.3$ & 1.1893 \\
\hline \multicolumn{8}{|c|}{ Power law } \\
\hline Object & $\lambda(\mathrm{mm})$ & $i\left(^{\circ}\right)$ & $\mathrm{PA}\left({ }^{\circ}\right)$ & $R_{\text {out }}(\mathrm{AU})$ & $p$ & $\Sigma_{40}\left(\mathrm{~g} \mathrm{~cm}^{-2}\right)$ & $\chi_{r}^{2}$ \\
\hline \multirow[t]{2}{*}{ DG Tau } & 1.3 & $27 \pm 9$ & $120 \pm 24$ & $72.3 \pm 4.0$ & $1.06 \pm 0.18$ & $35.7 \pm 3.6$ & 1.0611 \\
\hline & 2.8 & $32 \pm 11$ & $144 \pm 19$ & $81.8 \pm 9.3$ & $0.74 \pm 0.24$ & $32.1 \pm 4.5$ & 1.0629 \\
\hline RYTau & 1.3 & $66 \pm 2$ & $24 \pm 3$ & $70.5 \pm 3.9$ & $0.11 \pm 0.18$ & $9.7 \pm 1.2$ & 1.0897 \\
\hline$\ldots$ & 2.8 & $71 \pm 5$ & $20 \pm 4$ & $76.7 \pm 12.6$ & $0.68 \pm 0.51$ & $8.3 \pm 2.3$ & 1.1893 \\
\hline
\end{tabular}

Note. The uncertainties correspond to a likelihood of $99.7 \%$ (i.e., $3 \sigma$ ) for the normal distributions shown in Figures 5 and 6.

masses of about 1331 and $216 M_{\oplus}$ for DG Tau and RY Tau, respectively, by assuming a maximum grain size of $10 \mathrm{~cm}$ and a slightly different grain composition. Additional uncertainties in the disk mass come from the dust chemical composition. As discussed in the Appendix, the presence of ice or vacuum in the grains leads to smaller dust opacities at millimeter wavelengths 

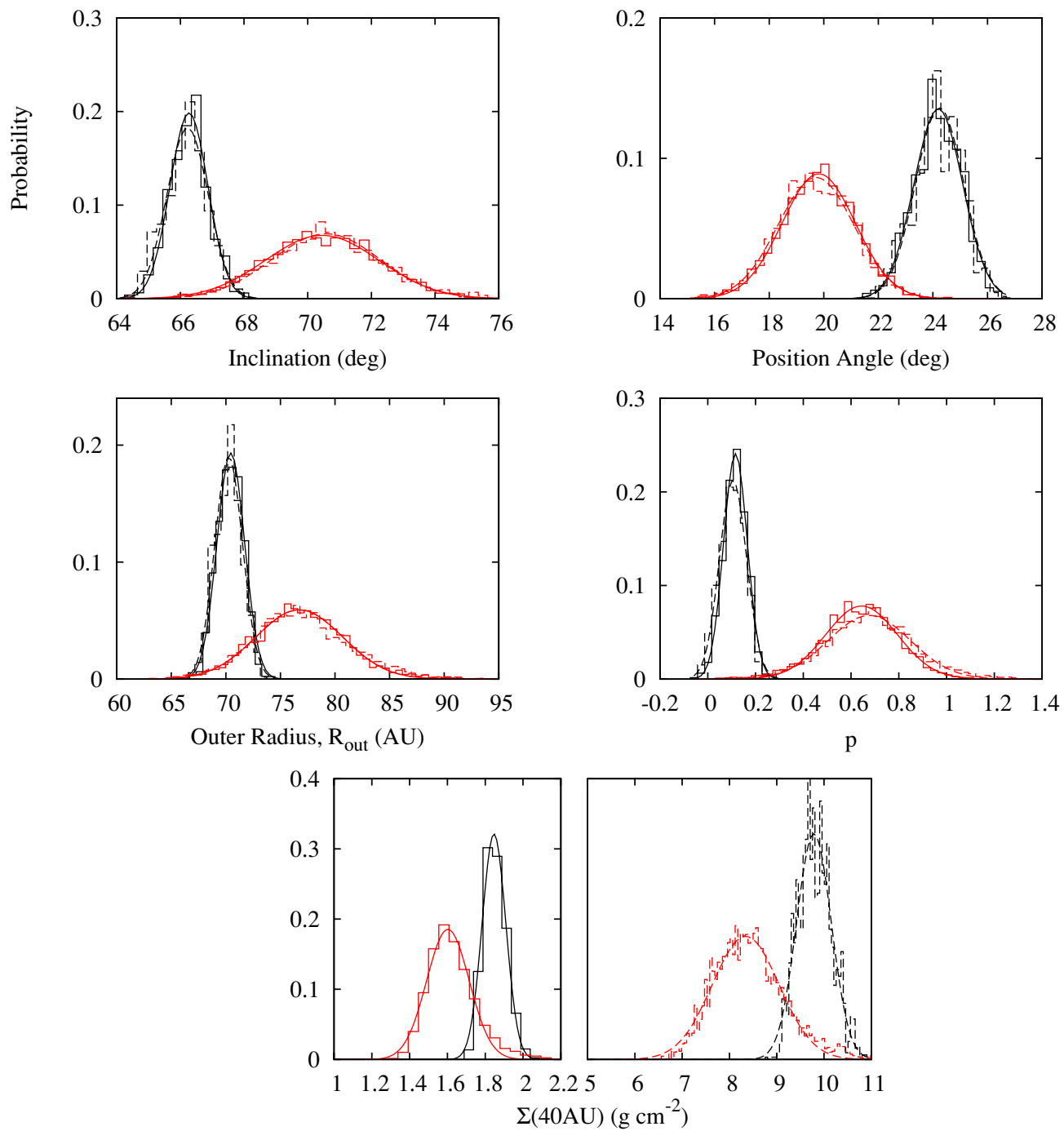

Figure 5. Probability distribution of the disk parameters obtained by fitting the RY Tau observations at $1.3 \mathrm{~mm}$ and $2.8 \mathrm{~mm}$ with a power-law surface density distribution. Colors, solid curves, and dashed curves are the same as in Figure 4.

(A color version of this figure is available in the online journal.)

and consequently produces higher disk masses. We therefore estimate that the circumstellar disks around DG Tau and RY Tau contain a minimum mass of dust of 30 and $10 M_{\oplus}$, respectively, while the upper limit is not constrained due to the uncertainties on the grain size distribution. Assuming the standard dust/gas ratio of 0.01 , these values correspond to minimum disk masses of 0.009 and $0.003 M_{\odot}$ for the DG Tau and RY Tau, respectively.

\subsection{Constraints on the Surface Density: Similarity Solution versus Power Law}

Figure 8 shows the comparison between models and observations in terms of the real part of the correlated flux as a function of the baseline length. To correct for the disk inclination, we deprojected the baseline assuming the inclinations and position angles listed in Table 3. In this figure, the results for $H$ and $L$ dust models lead to indistinguishable curves. Similarity solution and power-law models are represented with solid and dashed curves, respectively, and the observations are shown by black dots with error bars. It is clear that both the similarity solution and powerlaw disk models provide satisfactory fits to the observations. The similarity solution model provides smaller values of $\chi^{2}$ (see Tables 3 and 4) and, in the case of DG Tau, a better fit to the observations between 400 and $800 \mathrm{k} \lambda$. In this range of spatial frequencies, the power-law solution is characterized by a wiggle due to the sharp truncation of the dust emission at $72 \mathrm{AU}$. On the other hand, the exponential tapering of the similarity solution leads to a smooth visibility profile that matches extremely well the observations. The same behavior is present in the lower panel which compares the model and the observations at $2.8 \mathrm{~mm}$. However, in this case the observations at $B_{u v}>400 \mathrm{k} \lambda$ are too to distinguish between the two models. Although not conclusive, this result make the similarity solution model a more appealing explanation for the dust emission in circumstellar disks, confirming the conclusions of Hughes et al. (2008).

Figure 9 shows the surface density derived from the $1.3 \mathrm{~mm}$ observations for both the power law and the similarity solution model in the case of high dust opacity. The two models lead to similar values of $\Sigma(R)$ in the region where most of the $1.3 \mathrm{~mm}$ flux is emitted, namely between $\sim 15$ and $50 \mathrm{AU}$. In this region, the surface density in the RY Tau disk is almost constant with the radius, while it decreases roughly as $1 / R$ in the case of DG Tau. Inside $15 \mathrm{AU}$ and outside $50 \mathrm{AU}$, the observations lack both 

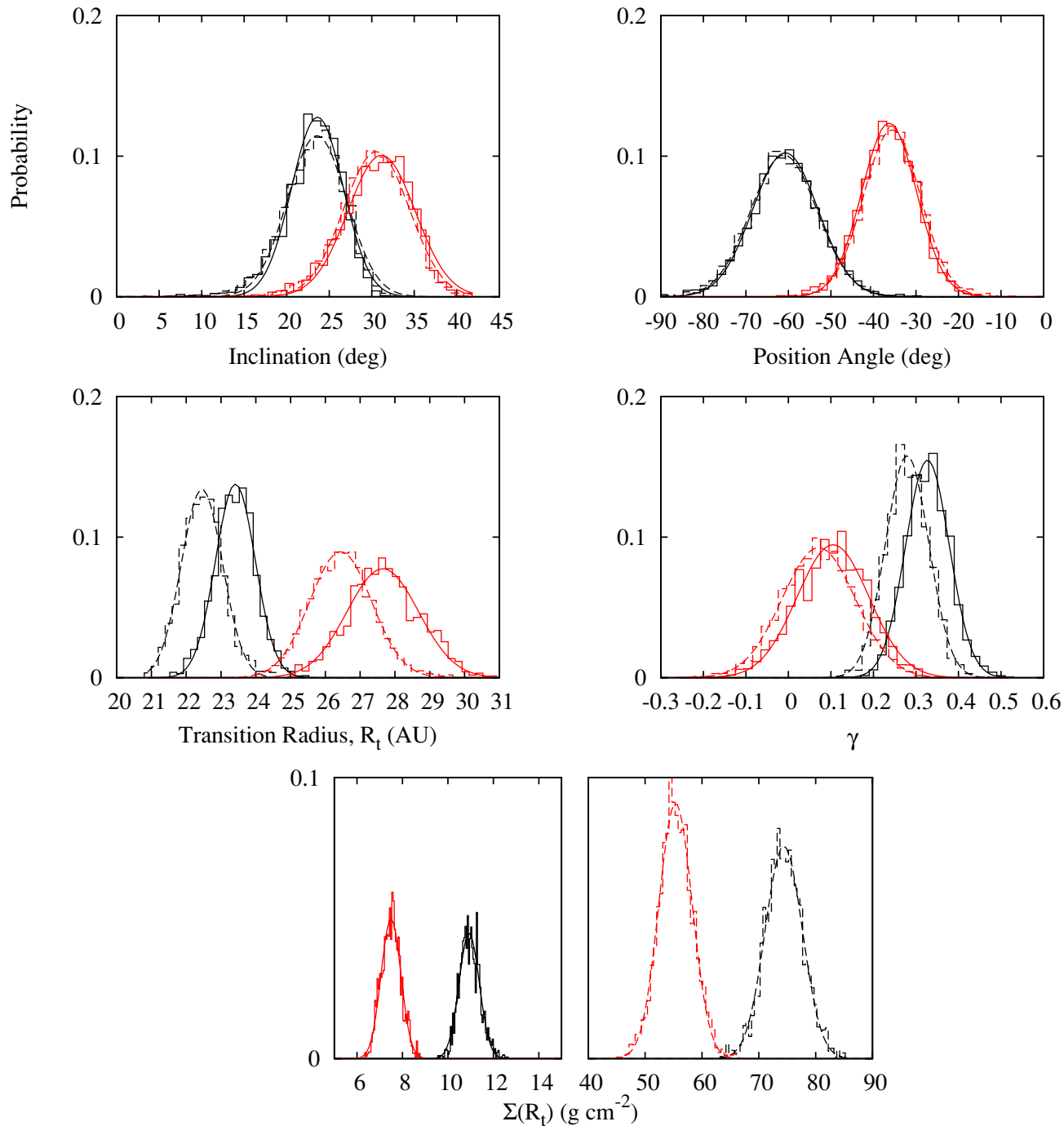

Figure 6. Probability distribution of the disk parameters obtained by fitting the DG Tau observations at $1.3 \mathrm{~mm}$ and $2.8 \mathrm{~mm}$ with the similarity solution for the surface density distribution. Colors, solid curves, and dashed curves are the same as in Figure 4.

(A color version of this figure is available in the online journal.)

the angular resolution and the sensitivity required to directly constrain the surface density. As a consequence, the values of $\Sigma(R)$ strongly depend on the assumed model and can differ by an order of magnitude at the disk inner radius.

\subsection{Surface Density and Implication on the Existence of Planets}

In this section, we discuss the implications of the inferred surface density on the presence of planets. The analysis is limited to surface density profiles obtained by fitting the observations at $1.3 \mathrm{~mm}$, which have the highest angular resolution.

\subsubsection{DG Tau}

For the similarity solution model, the surface density has a radial profile characterized by $\gamma \sim 0.31 \pm 0.18$ and $R_{t} \sim 23 \pm$ 2 AU. The transition radius $R_{t}$ agrees well with our earlier observations $(21 \pm 3)$ but $\gamma$ is significantly larger than the value of $-0.5 \pm 0.6$ from Paper I. The discrepancy is probably due to the fact that the earlier observations were taken in poorer weather conditions and the model fitting did not account for the atmospheric seeing. Figure 10 shows the residuals after subtracting the best-fit model to the new observations. Note that the power-law model gives very similar residuals. The residuals are as high as $3 \sigma-6 \sigma$ and are found at angular scales larger than 0 '.7 where the emission is slightly asymmetric (see Section 3). In this outermost disk region, the surface density may deviate significantly from the symmetric radial profile assumed in the model. We calculate that variations of $\pm 10-30 \mathrm{~g} \mathrm{~cm}^{-2}$ with respect to the best-fit surface density profile over a spatial region comparable with the beam size may produce the observed residuals. Larger variations of the surface density on smaller angular scales are also possible.

The residuals do not show global deviations from the smooth surface density profile, apparently excluding the possibility of gaps in the dust distribution that might be produced by a planet. Of course, low-mass planets may not produce any discernible gap and may still exist in the DG Tau disk. The formation of a gap is possible only if the efficiency in removing the material close to the planet orbital radius via tidal torques is larger than the mass accretion rate due to the disk viscosity (see, e.g., Lin \& Papaloizou 1993). If we assume the $\alpha$ prescription for the disk viscosity (Shakura \& Sunyaev 1973), and call $h$ 

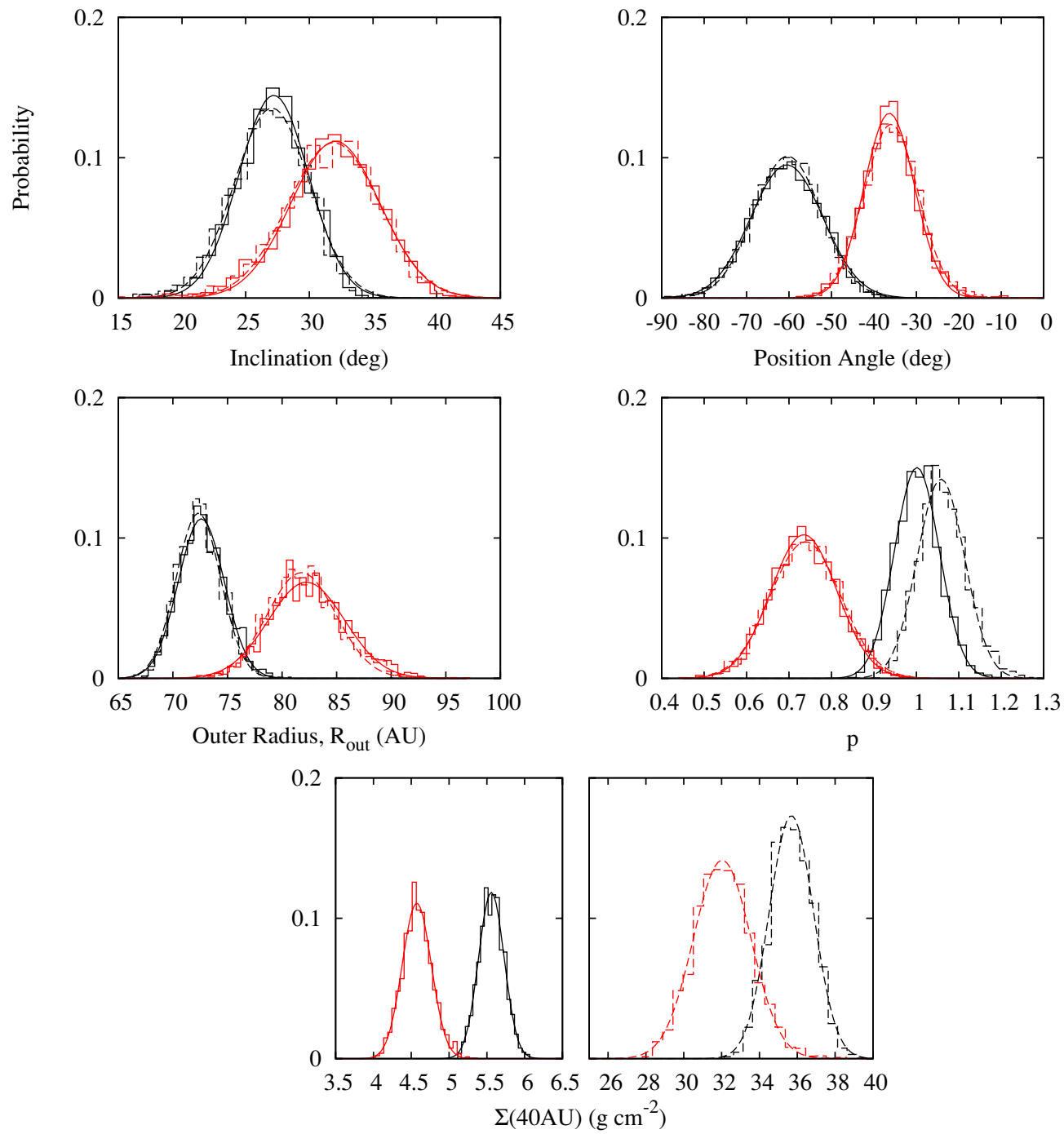

Figure 7. Probability distribution of the disk parameters obtained by fitting the DG Tau observations at $1.3 \mathrm{~mm}$ and $2.8 \mathrm{~mm}$ with a power-law surface density distribution. Colors, solid curves, and dashed curves are the same as in Figure 4.

(A color version of this figure is available in the online journal.)

the pressure scale height of the disk, a planet orbiting at radius $R_{p}$ can open a radial gap in the disk surface density only if $M_{p} / M_{\star}>32 \alpha\left(h / R_{p}\right)^{2}$ (Lin \& Papaloizou 1993; Bryden et al. 1999). Moreover, for a disk in hydrostatic equilibrium with the gravitational field of the central star, the pressure scale $h$ is proportional to $T_{i}\left(R_{p}\right)^{1 / 2} R_{p}^{3 / 2} M_{\star}^{-1 / 2}$ (Chiang \& Goldreich 1997). Since the temperature is $T_{i}\left(R_{p}\right) \propto R_{p}^{-1 / 2}$ (see Paper I for more details), the formation of a gap requires

$$
M_{p}>26.3 R_{p}^{1 / 2} \alpha
$$

where $M_{p}$ is expressed in Jupiter masses, $R_{p}$ in $\mathrm{AU}$, and the numerical constant is calculated for a disk temperature of $194 \mathrm{~K}$ at $1 \mathrm{AU}$ as determined from our disk model. Typical values of $\alpha$ are in the range $10^{-2}$ to $10^{-3}$, and imply that a planet can open a gap at $1 \mathrm{AU}$ only if its mass is larger than about $0.1 M_{J}$. To open a gap at $30 \mathrm{AU}$, the mass must be larger than about $0.5 M_{J}$.

To investigate the effects that a planet more massive than $0.1 M_{J}$ might have on the observations of the dust continuum emission, we simulated the presence of a planet in the DG Tau disk by opening a gap in the surface density distribution corresponding to the best-fit models discussed above. For simplicity, we assumed that the planet describes a circular orbit and that the gap can be represented by a circular ring. To be compatible with numerical simulations of planet-disk interaction, the half-width of the ring $\Delta$ is assumed to be equal to twice the Hill radius $R_{H}=R_{p} \sqrt[3]{M_{P} /\left(3 M_{\star}\right)}$ (e.g., Bryden et al. 1999; Wolf et al. 2007). In the region between $R_{p} \pm \Delta$, the surface density is depleted by a fraction $f$ that depends on the mass of the planet and on the disk viscosity. For $\alpha=10^{-3}$, we can assume $f=0$ for planet masses $M_{p}>1 M_{J}, f=0.1$ for $M_{p}=0.5 M_{J}, f=0.17$ for $M_{p}=0.3 M_{J}$, and $f=0.6$ for $M_{p}=0.1 M_{J}$ (Wolf et al. 2007). Therefore, only planets more massive than $1 M_{J}$ will produce completely cleaned gaps.

We simulated gaps corresponding to planets in the mass range $0.3-5 M_{J}$ and with orbital radii between 1 and 90 AU. For each model, we calculated the residuals as the difference between the observations of DG Tau at $1.3 \mathrm{~mm}$ and the model image. If the gap is too small compared to our angular resolution, or too faint compared with our sensitivity, the residuals will be similar to the case without gaps shown in Figure 10. In this case, we say that the gap is not detected. On the other hand, a 

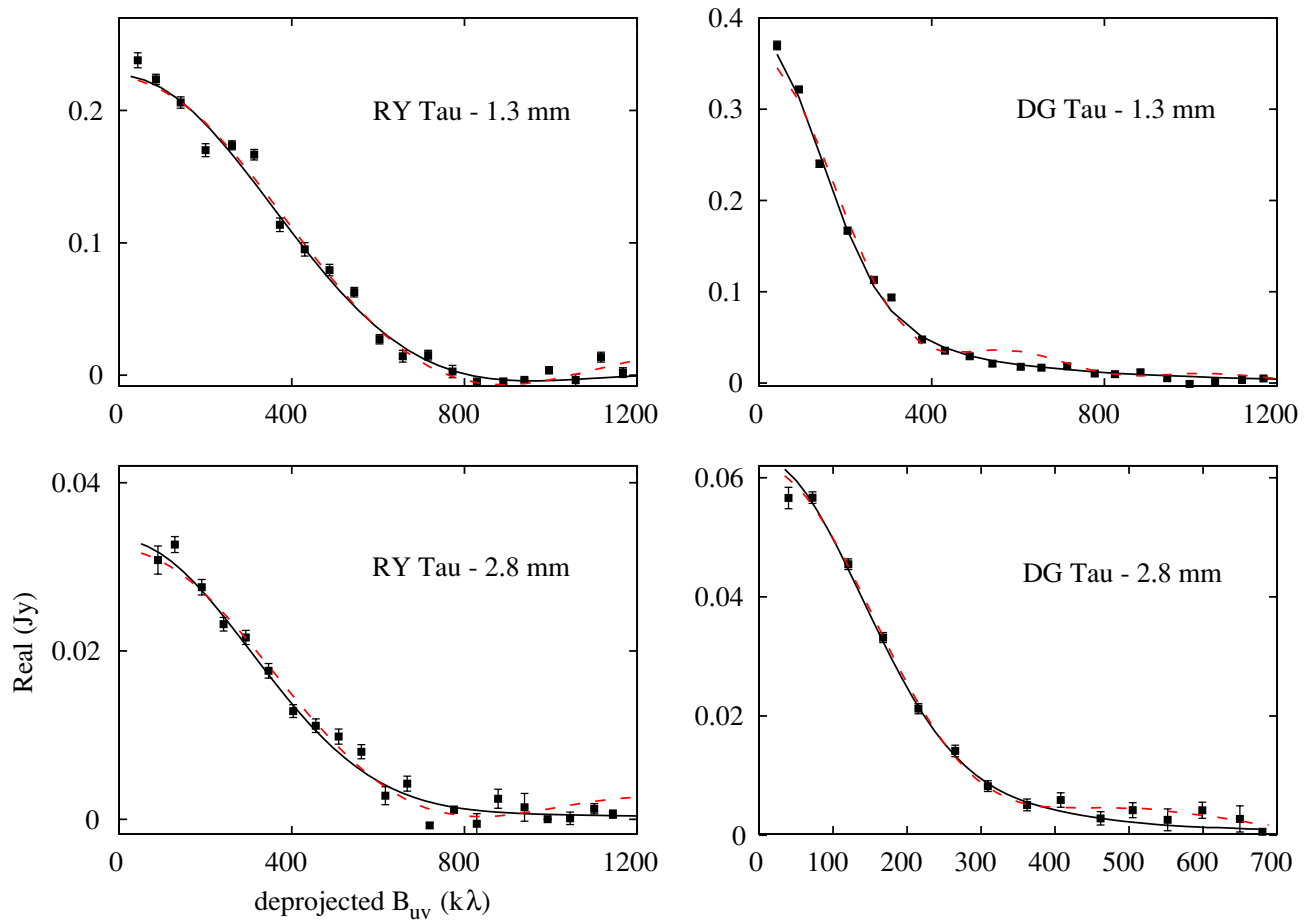

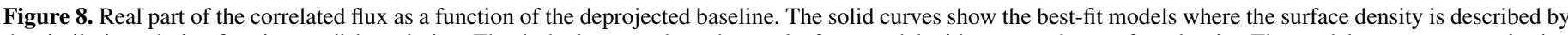

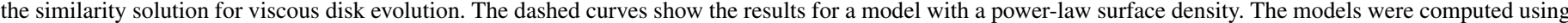
the high dust opacity, but identical results were obtained for the low dust opacity case.

(A color version of this figure is available in the online journal.)

large and deep gap will produce a bright ring in the residual. To quantify how reliable the detection of a gap is, we define a signal-to-noise ratio of the gap (gap S/N) in the following way. First, we deproject the residual for the inclination and position angle of the DG Tau disk. Then we take the radial average of the residuals at the distance corresponding to the orbital radius of the planet adopting a radial bin width equal to the FWHM of the synthesized beam (i.e., $0^{\prime \prime} 17$ ). We define the gap $\mathrm{S} / \mathrm{N}$ as the mean residual in the radial bin divided by the uncertainty in the mean. In this way, detected gaps correspond to gap $\mathrm{S} / \mathrm{N}>3$.

The results are summarized in the upper panel of Figure 11. Planets with masses and radii that lead to gap $\mathrm{S} / \mathrm{N}>3$ produce detectable gaps. No gaps are detected in our observations of the DG Tau disk at more than $3 \sigma$ (see the red curve). This enables us to constrain the masses and orbital radii of any planets that may be present. In particular, we can exclude that planets more massive than Jupiter exist between 5 and 40 AU, or that planets with masses slightly smaller than Jupiter exists between 10 and 25 AU. The observations lack both the angular resolution and the sensitivity required to detect gaps produced by planets with a mass smaller than about $0.5 M_{J}$.

An important caveat is that a planet may exist but may not have had enough time to completely open a gap in the disk. The gap formation timescale $\tau_{\Delta}$ results from the tradeoff between the efficiency of the tidal torque exercised by the planet in removing angular momentum, and the accretion of new material coming from larger radii in the gap due to the disk viscosity. A lower limit of the gap formation timescale is obtained in the zero viscosity limit. In this case, an analytic formulation is provided by Bryden et al. (1999) in the form

$$
\tau_{\Delta}^{\min } \simeq \frac{P}{q^{2}}\left(\frac{\Delta}{R_{p}}\right)^{5}
$$

where $P$ is the orbital period, $q=M_{p} / M_{\star}$, and $\Delta=2 R_{H}$ as defined above. Assuming Keplerian rotation, we can rewrite the timescale for the gap formation as

$$
\tau_{\Delta}^{\min }=1.1 \mathrm{Myr} \times\left(\frac{M_{\star}}{M_{\odot}}\right)^{3 / 2}\left(\frac{R_{p}}{\mathrm{AU}}\right)^{3 / 2}\left(\frac{M_{p}}{M_{J}}\right)^{-2}\left(\frac{\Delta}{R_{p}}\right)^{5} .
$$

The upper panel of Figure 12 shows the calculated values of $\tau_{\Delta}^{\min }$ for the stellar mass of DG Tau $\left(0.3 M_{\odot}\right)$. In the case of a planet with a mass between 0.3 and $0.5 M_{J}$ orbiting at a radius larger than $40 \mathrm{AU}$, the minimum timescale for the gap formation is comparable with the age of the system $(0.1 \mathrm{Myr})$. For more massive planets, or for closer radii, the minimum gaps timescale is a small fraction of the age of the system.

We conclude that, for DG Tau, the observations lack the sensitivity and angular resolution required to investigate the presence of planets less massive than about $0.5 M_{J}$ at any orbital radius. Our analysis indicates that no planets more massive than Jupiter are present between 5 and 50 AU, unless they are younger than $10^{4}$ years.

\subsection{2. $R Y$ Tau}

The similarity solution for the disk surface density is characterized by $\gamma=-0.56 \pm 0.18$ and $R_{t} \sim 26 \pm 3 \mathrm{AU}$. As shown in Figure 9, the surface density increases roughly as $\sqrt{R}$ from the inner radius at $0.1 \mathrm{AU}$ up to about $26 \mathrm{AU}$ and then decreases exponentially outward. This supports the suggestion in Section 3 that the RY Tau inner disk might be partially dust depleted with respect to power-law disk models. We note that this surface density profile may provide an explanation for both the double peak intensity at $1.3 \mathrm{~mm}$ and the disk excess at infrared wavelengths. Indeed, within $10 \mathrm{AU}$ the model disk remains optically thick at optical and infrared wavelengths, exhibiting the infrared excess typical of classical disks. 

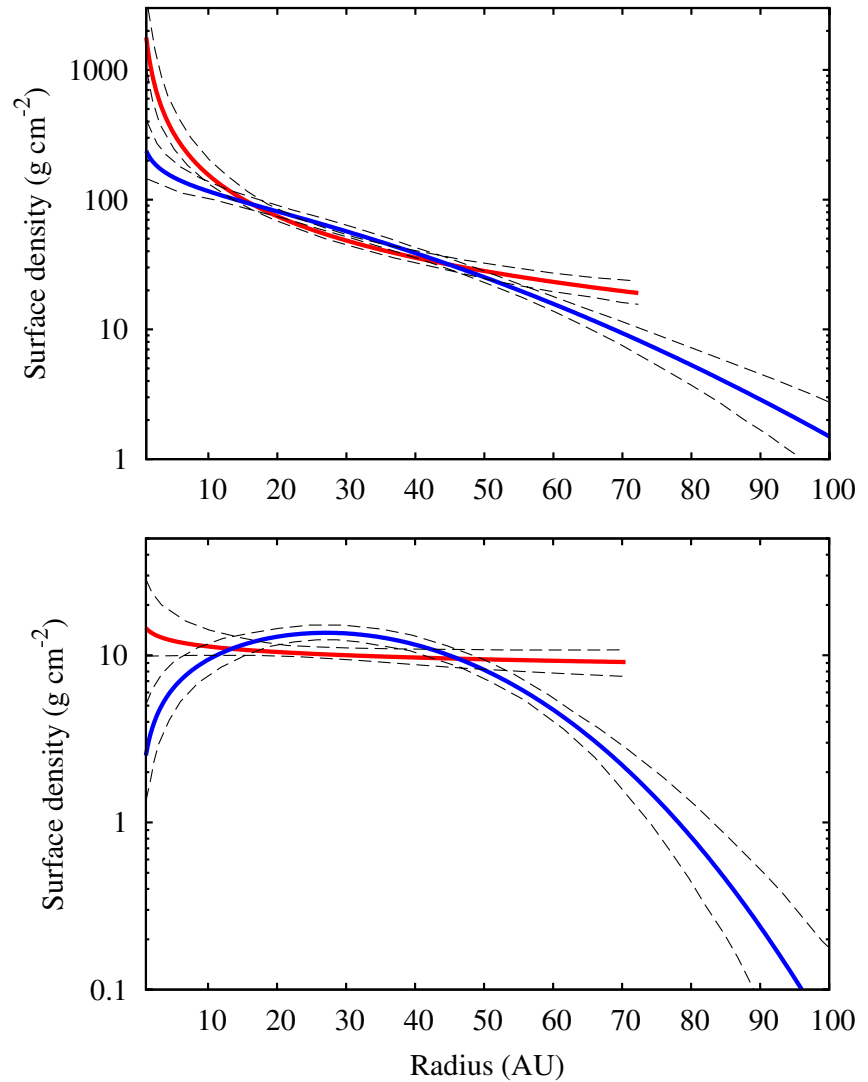

Figure 9. Surface density for DG Tau (top panel) and RY Tau (lower) disks. The red and blue curves show the best-fit solutions for the power law and the similarity solution models, respectively, in the low opacity case $L$. The dashed curves show the $3 \sigma$ uncertainty range for the surface density. The surface densities for the high opacity case will have similar shapes, but will be about a factor of 6 lower.

(A color version of this figure is available in the online journal.)

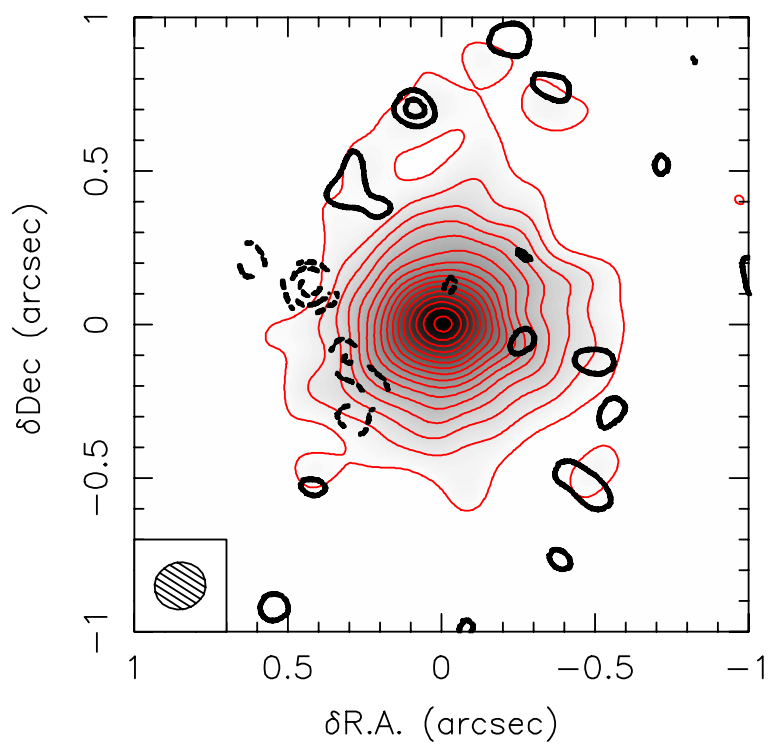

Figure 10. Black curves show the residuals for the $1.3 \mathrm{~mm}$ DG Tau observations after subtracting the best-fit model for the similarity solution. Contours start at $3 \sigma$ and are spaced by $1 \sigma$. The thin red curves and the color scale show the observed dust emission, with contours spaced by $3 \sigma$.

(A color version of this figure is available in the online journal.)

At larger radii, the surface density in the RY Tau disk decreases smoothly and the residuals calculated by subtracting
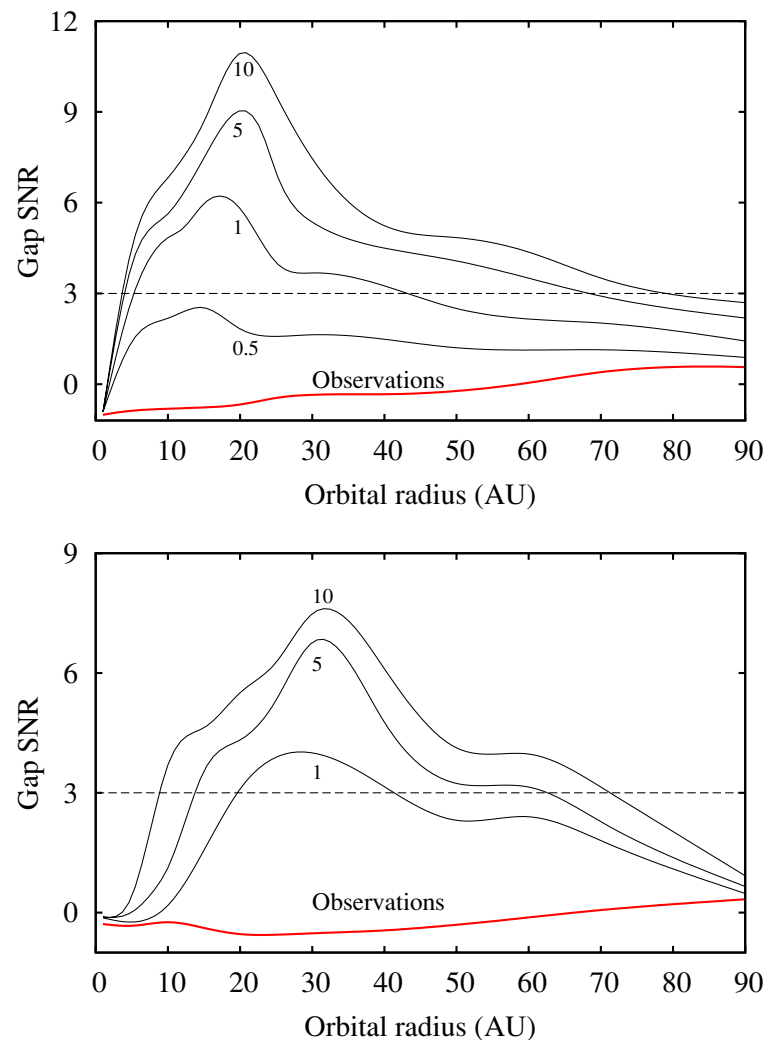

Figure 11. $\mathrm{S} / \mathrm{N}$ of the detection of a gap generated by a planet as a function of the orbital radius $R_{p}$ and the planet mass. The different curves correspond to masses between 0.5 and $10 M_{J}$ as labeled. The thick red curves indicate the $\mathrm{S} / \mathrm{N}$ measured from the $1.3 \mathrm{~mm}$ images after subtracting the best-fit model for the similarity solution. For gap $\mathrm{S} / \mathrm{N}>3$, planets should produce a detectable gap. The upper and lower panels refer to the case of DG Tau and RY Tau, respectively.

(A color version of this figure is available in the online journal.)

the best-fit models to the $1.3 \mathrm{~mm}$ dust emission map do not show any structure at more than $3 \sigma$. This excludes strong deviations from an unperturbed viscous disk profile.

The lower panel of Figure 11 shows the $\mathrm{S} / \mathrm{N}$ of the detection of a gap generated by planets of 1,5 , and $10 M_{J}$ as a function of the orbital radius. Due to the higher disk inclination and stellar mass, a planet orbiting around RY Tau would produce a less visible gap. In particular, our observations seem to exclude the presence of planets more massive than $5 M_{J}$ between 10 and $60 \mathrm{AU}$. Given the higher stellar mass of RY Tau, the minimum timescale for the formation of gaps is an order of magnitude larger than the case of DG Tau (see the lower panel of Figure 12). This implies that planets less massive than Jupiter orbiting at more than about $30 \mathrm{AU}$ may not have had enough time to form a gap in the disk.

\subsection{Radial Dependence of the Dust Properties}

A comparison of the best-fit solutions obtained for the wavelengths of $1.3 \mathrm{~mm}$ and $2.8 \mathrm{~mm}$ enables us to investigate the dependence of dust opacity on the orbital radius. If the dust opacity is constant throughout the disk as assumed in Section 4, the model fitting necessarily leads to the same surface density profile for observations at two different wavelengths. Otherwise, different $\Sigma(R)$ would suggest a radial variation in the relative dust opacities at the observed wavelengths. To understand this point, we assume that the dust emission is optically thin. In this case the observations constrain the product $\Sigma_{\lambda}(R) \times k_{\lambda}$, where 

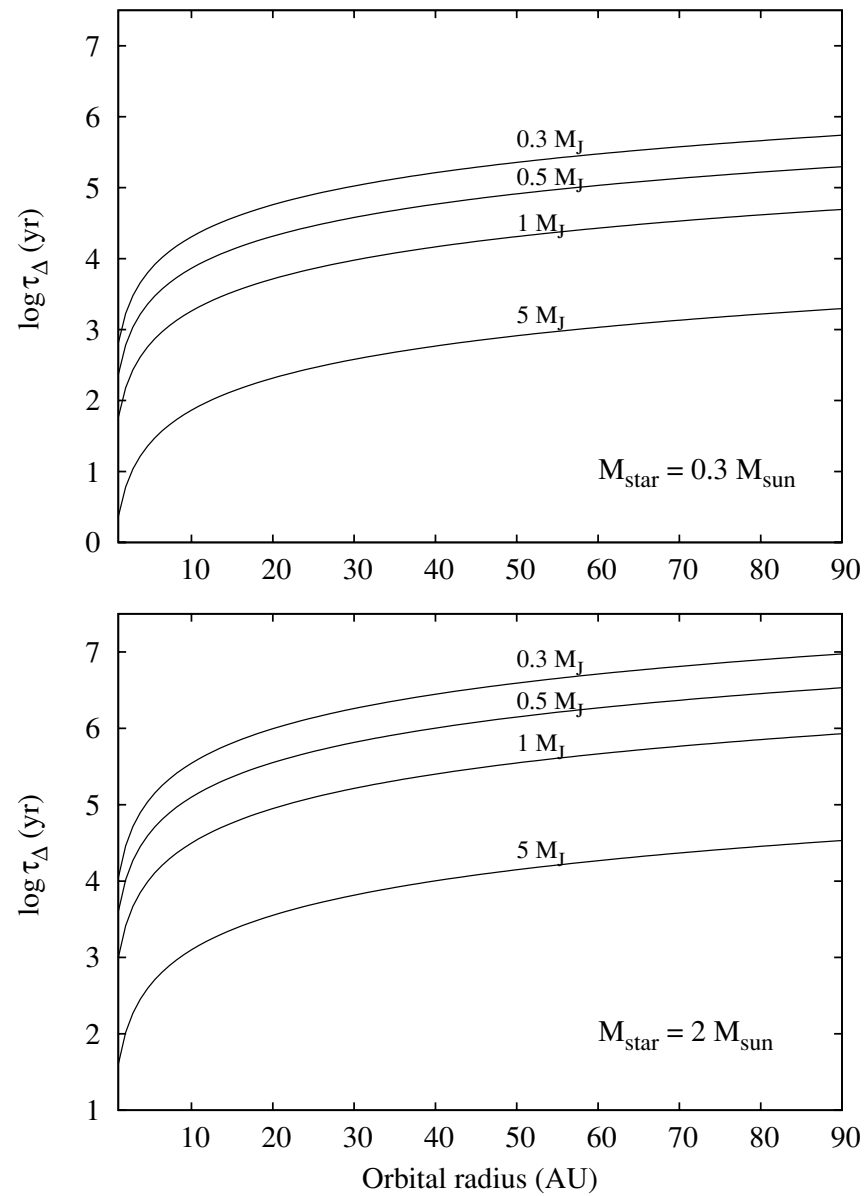

Figure 12. Minimum timescale $\left(\tau_{\Delta}\right)$ for the formation of a gap as a function of the orbital radius and mass of the planet. The different curves correspond to planet masses between 0.3 and $5 M_{J}$ as labeled in the figure. The upper and lower panels show the timescale for a central star of $0.3 M_{\odot}$ and $2 M_{\odot}$, respectively.

$\Sigma_{\lambda}(R)$ is the surface density obtained by fitting the observations at the wavelength $\lambda$. In the more general case in which the dust opacity depends on the orbital radius, we can write

$$
\Sigma_{\lambda}(R) \times k_{\lambda}=\tilde{\Sigma}(R) \times \tilde{k}_{\lambda}(R) .
$$

The left side of this equation contains the opacity discussed in Section 4 and the surface density derived from the model fitting. The right side contains the unknown "true" surface density $\tilde{\Sigma}(R)$ in the case in which the "true" dust opacity $\tilde{k}_{\lambda}(R)$ varies with the radius. The ratio of Equation (6) for two different wavelengths $\lambda_{0}$ and $\lambda_{1}$ leads to

$$
\frac{\Sigma_{\lambda_{0}}(R)}{\Sigma_{\lambda_{1}}(R)} \times \frac{k_{\lambda_{0}}}{k_{\lambda_{1}}}=\frac{\tilde{k}_{\lambda_{0}}(R)}{\tilde{k}_{\lambda_{1}}(R)}=\left(\frac{\lambda_{1}}{\lambda_{0}}\right)^{\beta(R)} .
$$

Here we assumed that at each radius the dust opacity can be expressed by a power-law $k_{\lambda} \propto \lambda^{-\beta}$. Finally, taking the logarithm of this latter equation we can write

$$
\beta(R)=\beta_{c}+\Delta \beta(R),
$$

where $\beta_{c}=\log \left(k_{\lambda_{1}} / k_{\lambda_{0}}\right) / \log \left(\lambda_{0} / \lambda_{1}\right)$ and $\Delta \beta(R)$ has the form

$$
\Delta \beta(R)=\log ^{-1}\left(\frac{\lambda_{1}}{\lambda_{0}}\right) \times \log \left[\frac{\Sigma_{\lambda_{0}}(R)}{\Sigma_{\lambda_{1}}(R)}\right] .
$$

If $\Sigma_{\lambda_{1}}=\Sigma_{\lambda_{0}}$, the dust opacity slope is constant throughout the disk and assumes the value discussed in Section 4. Otherwise, we can use the latter equation to investigate the radial variation of $\beta$.

The best-fit solutions for $\Sigma(R)$ obtained at $1.3 \mathrm{~mm}$ and $2.8 \mathrm{~mm}$ are shown in Figures 5 and 6 with black and red curves, respectively. The best-fit parameters are summarized in Tables 3 and 4 . The quoted uncertainties correspond to a likelihood of $99.7 \%$ (i.e., $3 \sigma$ ) and are calculated by fitting a normal distribution to the measured probability distributions. For RY Tau, the disk model obtained by fitting the two wavelengths separately are in agreement within $3 \sigma$. For DG Tau the solutions disagree by more than $3 \sigma$ only in the case of the similarity solution and high dust opacity.

Figure 13 shows the radial variation of $\beta$ as defined in Equation (8) for both DG Tau and RY Tau. The region marked with color indicates values of $\beta$ within $3 \sigma$ from the radial profile corresponding to the best-fit solution for the surface density in the case of the similarity solution model. Values of $\beta$ outside this region are rejected by our observations.

The results for RY Tau and DG Tau are clearly consistent with a large variety of radial profiles of $\beta$. For both sources, $\beta$ is better constrained between radii of 20 and $70 \mathrm{AU}$. However, even in this interval, the observations constrain possible variation of $\beta$ to within only $\Delta \beta<0.7$. Nevertheless, across most of the disk the circumstellar dust differs from that observed in the interstellar medium (ISM). Dust in the ISM is characterized by sub-micron dust grains and by a millimeter opacity slope of $\beta \sim 1.7$. In contrast, in both sources, $\beta$ is smaller than 1.7 up to at least $80 \mathrm{AU}$, suggesting that the circumstellar dust has been processed and, in particular, has increased its size up to a maximum value that varies between $20 \mu \mathrm{m}$ and a few centimeters.

Although in both sources $\beta$ may be constant throughout the disk (see dashed lines), our results suggest that $\beta$ decreases with the radius in the DG Tau disk. As discussed in Section 4, $\beta$ depends on a number of poorly constrained quantities, such as composition, structure, and size of the dust grains. For example, varying $\beta$ from $\sim 1$ to $\sim 0.2$, similar to what is suggested for DG Tau between 10 and $60 \mathrm{AU}$, may be due to the maximum grain size increasing from $20 \mu \mathrm{m}$ to $1 \mathrm{~cm}$ for $q=3$, or, alternatively, to a decrease of $q$ from 4 to 3 if the maximum grain size is between 1 and $10 \mathrm{~cm}$. In short, the interpretation of $\beta$ only in terms of the grain size distribution can be very misleading. It seems most plausible that both dust composition and the relative contributions of smaller and larger grains change through the disk, contributing to the variation of the dust opacity. It is clear that better constraints on the radial profile of $\beta$ are required before pushing the investigation further.

In this regard, we note that the current constraints on the radial variation of $\beta$ are limited by two factors. First, although the angular resolution of the observations described here is significantly better than hitherto possible, the dust surface density is well constrained only between 15 and $50 \mathrm{AU}$, where most of the observed flux is emitted (see the discussion in Section 5.3). At smaller and larger radii, the surface density is uncertain by almost an order of magnitude. Second, our analysis is hampered by the small separation in wavelength between the observations since $\Delta \beta(R)$ is proportional to $\log ^{-1}\left(\lambda_{1} / \lambda_{0}\right)$. The uncertainties shown in Figure 13 can be reduced by a factor of 2 by extending the observations at $7 \mathrm{~mm}$. These observations will become possible with the expanded correlator on the Expanded Very Large Array (EVLA). 

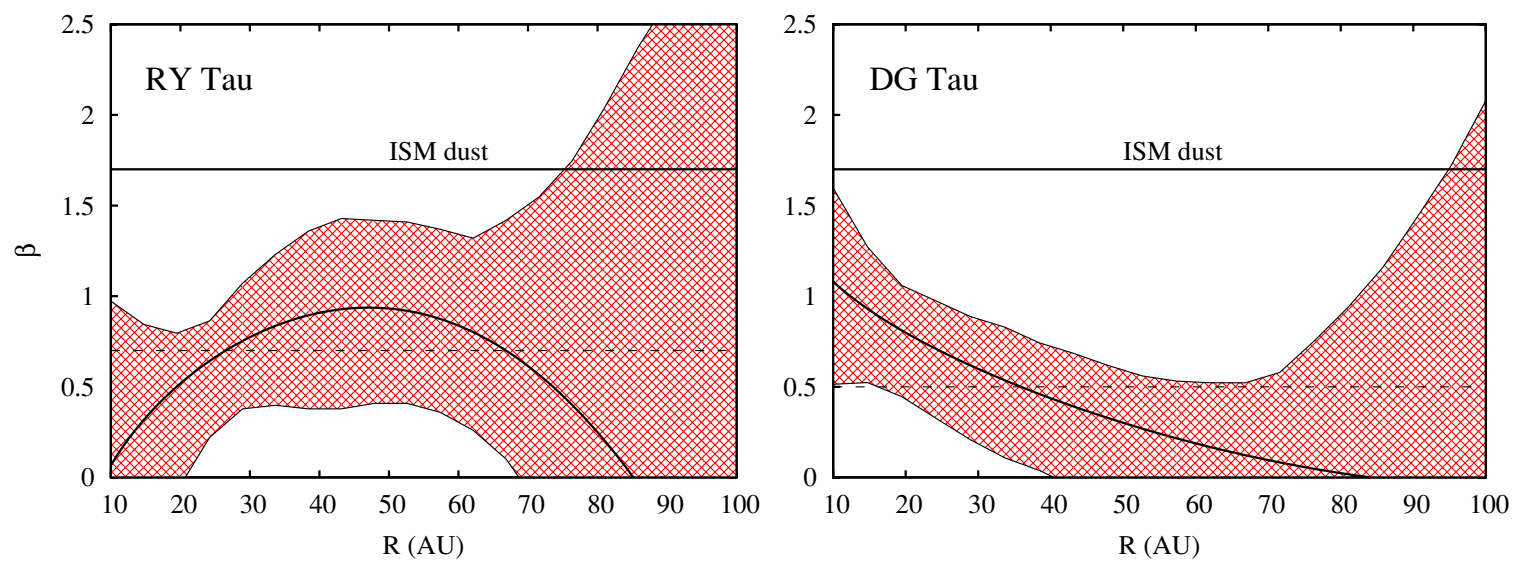

Figure 13. Slope of the millimeter dust opacity $\beta$ as a function of the radius for RY Tau (left panel) and DG Tau (right panel). Values of $\beta$ outside the colored region are excluded by our observations at more than the $3 \sigma$ level. The dashed lines show the mean value of $\beta$ derived in Paper I as discussed in Section 4 . The thick solid curve shows the radial profile of $\beta$ corresponding to the best-fit solution for the disk surface density at both 1.3 and $2.8 \mathrm{~mm}$. The solid horizontal line shows the value of $\beta$ characteristic of the dust in the ISM.

(A color version of this figure is available in the online journal.)

\section{CONCLUSIONS}

We have presented CARMA observations of the dust thermal emission at the wavelengths of $1.3 \mathrm{~mm}$ and $2.8 \mathrm{~mm}$ from the circumstellar disks around the pre-main-sequence stars RY Tau and DG Tau. The observations are characterized by unprecedented angular resolution of $\sim 0^{\prime} .15$ and 0.30 at $1.3 \mathrm{~mm}$ and $2.8 \mathrm{~mm}$, respectively, corresponding to spatial scales of 20 and $40 \mathrm{AU}$ at the distance of Taurus. Based on these images, we have addressed three fundamental questions related to the formation of planets in the disk around pre-main-sequence stars. What is the radial density distribution of circumstellar dust? Does the dust emission show any indication of the presence of (proto)-planets? Do the dust properties vary with orbital radius?

By analyzing the morphology of the surface brightness of the dust emission and comparing the observations with theoretical disk models, we make the following conclusions.

1. Both the classical power-law disk surface density (Hayashi 1981) and the similarity solution for the viscous evolution of a Keplerian disk (Hartmann et al. 1998) fit the observations well. The surface density is well constrained between 15 and $50 \mathrm{AU}$. In this region, the two models lead to values of $\Sigma$ that agree within $30 \%$ for a fixed dust opacity. At smaller and larger radii, the surface density depends on the assumed model and varies by almost an order of magnitude. We have verified that the assumptions on the dust opacity have a small effect on the model fitting and, therefore, on the radial profile of the dust density. However, the total disk mass may vary by almost 2 orders of magnitude for different dust compositions and grain size distributions.

2. The dust emission in DG Tau is mostly radially symmetric. It is characterized by a single, central peak and smoothly decreases up to an angular distance of about 0.5 . Theoretical disk models reproduce the observation very well, with randomly distributed residuals between $3 \sigma$ and $6 \sigma$. No systematic deviation from the similarity solution for the surface density of a viscous disk is observed. By simulating the presence of planets in the disk via the gap in the surface density produced by tidal torques, we find that the observations exclude the presence of planets more massive than Jupiter orbiting between 5 and $40 \mathrm{AU}$ from the central star, unless the planets are very young $\left(<10^{4} \mathrm{yr}\right)$ and have not had the time to open a gap in the disk. The observations lack both the angular resolution and sensitivity to investigate the presence of planets less massive than about $0.5 M_{J}$.

For RY Tau, the dust emission is characterized by two peaks separated by about 28 AU that suggest a decrease in the surface density, or dust opacity, within $14 \mathrm{AU}$ of the central star. We found that the similarity solution for the disk surface density is characterized by a negative value of $\gamma$, and provides a reasonable explanation for the double peak intensity observed at $1.3 \mathrm{~mm}$. Depletion of millimeter dust grains (Dullemond \& Dominik 2005), decreasing values of the disk viscosity, or the presence of planetesimals, may produce the observed dust morphology. At larger radii, the dust emission shows a very smooth profile with no asymmetries or gaps. The lack of gaps in the disk suggests that any planets between 10 and $50 \mathrm{AU}$ are less massive than about $5 M_{J}$, or, as for DG Tau, are very young.

3. The best-fit models to the $1.3 \mathrm{~mm}$ and $2.8 \mathrm{~mm}$ data were compared to investigate the radial dependence of the slope opacity $\beta$, assuming that the dust opacity at millimeter wavelengths is expressed by a power-law $k_{\lambda} \propto \lambda^{-\beta}$. We can exclude cases in which $\beta$ varies by more than 0.7 within 70 AU. Nevertheless, between 20 and 70 AU, the disks around DG Tau and RY Tau are characterized by values of $\beta$ that are smaller than those found in the ISM. This implies that the dust has been reprocessed and has grown in size up to a radius of at least $20 \mu \mathrm{m}$. The investigation of the radial variation of $\beta$ is still limited by the angular resolution and by the small separation in wavelength between the observations. In the future, the Atacama Large Millimeter/ submillimeter Array and EVLA will play crucial roles in the investigation of the radial dependence of the dust properties by increasing the angular resolution and the interval in wavelength.

We thank Antonella Natta and referee Steven Beckwith for reading the manuscript and providing helpful suggestions. We thank the OVRO/CARMA staff and the CARMA observers for their assistance in obtaining the data. Support for the Combined Array for Research in Millimeter Astronomy construction was derived from the Gordon and Betty Moore Foundation, the Kenneth T. and Eileen L. Norris Foundation, the Associates of 

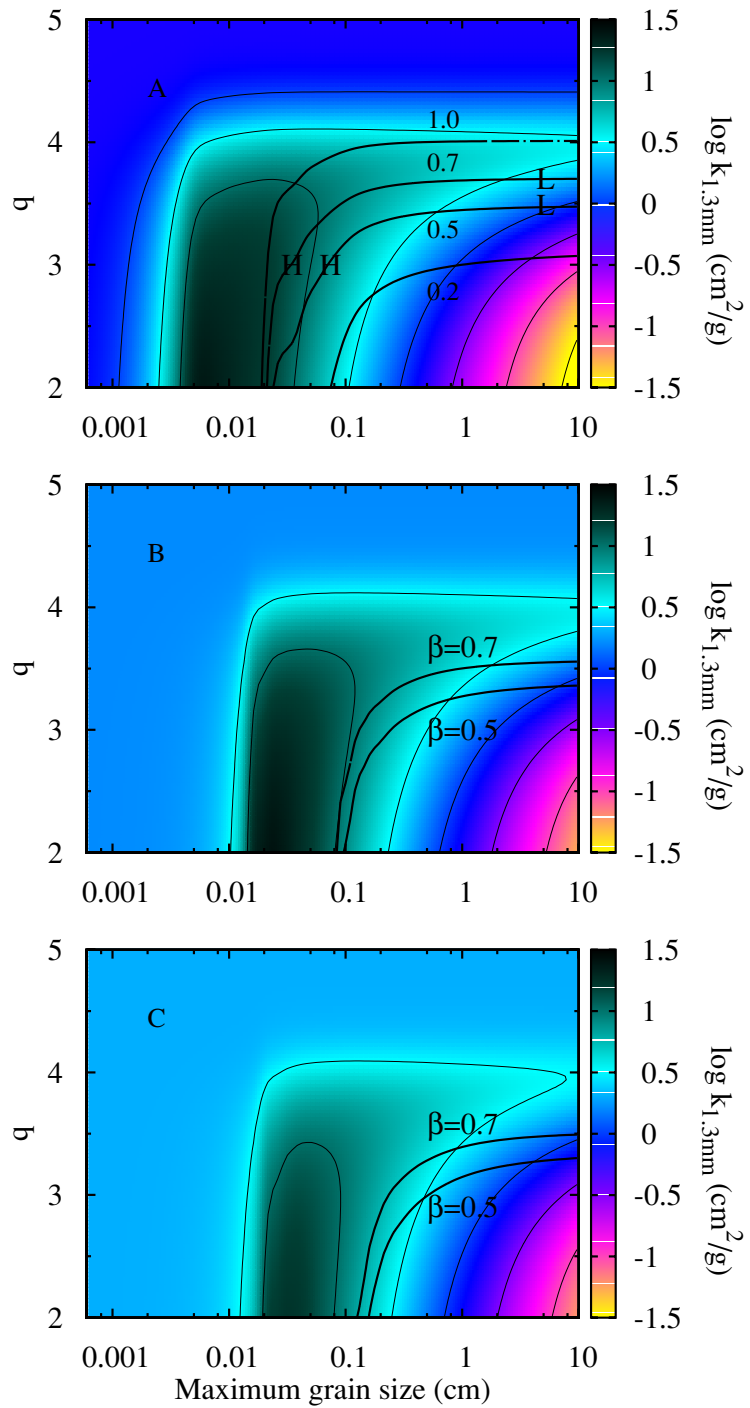

Figure 14. Color gradient depicts the dust opacity as a function of the maximum grain size $a_{\max }$ and the slope of the grain size distribution $q$. Results are shown for three different dust models (A, B, and C) as described in the text. The minimum grain size is fixed at $5 \times 10^{-7} \mathrm{~cm}$. Thin solid curves show the dust opacity contours and are spaced by 0.5 dex. The thick solid curves show the possible pairs of $\left(a_{\max }, q\right)$ that lead to values for the opacity slope $\beta$ equal to 0.5 (DG Tau) and 0.7 (RY Tau). The letters $H$ and $L$ indicate the high and low dust opacity models adopted in fitting the observed dust emission.

(A color version of this figure is available in the online journal.)

the California Institute of Technology, the states of California, Illinois, and Maryland, and the National Science Foundation. Ongoing CARMA development and operations are supported by the National Science Foundation under a cooperative agreement (grant AST 08-38260), and by the CARMA partner universities. This work was performed in part under contract with the Jet Propulsion Laboratory (JPL) funded by NASA through the Michelson Fellowship Program. JPL is managed for NASA by the California Institute of Technology.

\section{APPENDIX}

\section{EFFECTS OF THE ASSUMPTIONS ON THE DUST COMPOSITION ON THE DUST OPACITY AND SIZE}

The slope of the dust opacity $\beta$ measured at millimeter wavelengths has been widely adopted to constrain the size and opacity of the circumstellar dust (see, e.g., Ricci et al. 2010).
In this appendix, we investigate how these latter two quantities depend on the assumption on the grain size distribution and composition.

The color scale in Figure 14 shows the dust opacity at $1.3 \mathrm{~mm}$ calculated for three different dust compositions. In model A we assume that the grains are compact spheres composed of astronomical silicates and organic carbonates (Weingartner \& Draine 2001; Zubko et al. 1996). This is the dust model adopted in the paper. In model B the grain is composed of silicates, carbonates, and water ice. Finally, in model C the ice is replaced by a vacuum, resulting in a porous grain made of silicates and carbonates. We assume a mass ratio of 1 between silicates and organics, and of 0.7 between silicates and the water ice or the vacuum (Pollack et al. 1994). The resulting bulk densities are $2.5 \mathrm{~g} \mathrm{~cm}^{-3}$ for model A, $1.9 \mathrm{~g} \mathrm{~cm}^{-3}$ for model B, and $1.5 \mathrm{~g} \mathrm{~cm}^{-3}$ for model $\mathrm{C}$. We assume a grain size distribution $n(a) \propto a^{-q}$ and fix the minimum grain size to $0.005 \mu \mathrm{m}$. We then calculate the dust opacity by varying the maximum grain size $a_{\max }$ between $6 \times 10^{-4}$ and $10 \mathrm{~cm}$ and the slope $q$ between 2 and 5 .

The solid thick curves show the values of $\left(a_{\max }, q\right)$ required to obtain the values of $\beta$ measured for DG Tau and RY Tau, 0.5 and 0.7 , respectively. From the figure, it is clear that the value of $\beta$ sets a lower bound to the maximum grain radius in the grain size distribution (see, e.g., Natta et al. 2004). This lower bound is a function of the grain composition and increases by almost an order of magnitude between models A and C. For example, in order to have $\beta=0.7$, the maximum grain size must be at least $0.03 \mathrm{~cm}$ for compact grains in model A, or about $0.2 \mathrm{~cm}$ for the porous grains in model C. Note, however, that we can obtain the same value of $\beta$ with $a_{\max }=10 \mathrm{~cm}$ and $q$ between 3.2 and 3.7. Deriving the maximum grain size from the measure of $\beta$ is evidently strongly degenerate.

This introduces a large uncertainty on the dust opacity and ultimately on the total mass of circumstellar dust derived from millimeter observations. Even if we limit the analysis to the generally adopted value $q=3.5$ (see, e.g., Brauer et al. 2008), the dust opacity for $\beta=0.7$ varies from about $8.4 \mathrm{~cm}^{2} \mathrm{~g}^{-1}$ of dust in the case of the model A and $a_{\max }=0.1 \mathrm{~cm}$, to $1.2 \mathrm{~cm}^{2} \mathrm{~g}^{-1}$ of dust for model $\mathrm{C}$ and $a_{\max }=10 \mathrm{~cm}$, leading to disk masses that differ by almost an order of magnitude.

\section{REFERENCES}

Akeson, R. L., et al. 2005, ApJ, 635, 1173

Alexander, R. D., Clarke, C. J., \& Pringle, J. E. 2006, MNRAS, 369, 216

Andrews, S. M., Wilner, D. J., Hughes, A. M., Qi, C., \& Dullemond, C. P. 2009, ApJ, 700, 1502

Beckwith, S. V. W., \& Sargent, A. I. 1991, ApJ, 381, 250

Beckwith, S. V. W., Sargent, A. I., Chini, R. S., \& Guesten, R. 1990, AJ, 99, 924

Bertout, C., Robichon, N., \& Arenou, F. 1999, A\&A, 352, 574

Brauer, F., Dullemond, C. P., \& Henning, Th. 2008, A\&A, 480, 859

Brown, J. M., Blake, G. A., Qi, C., Dullemond, C. P., \& Wilner, D. J. 2008, ApJ, 675,109

Brown, J. M., Blake, G. A., Qi, C., Dullemond, C. P., \& Wilner, D. J. 2009, ApJ, 704, 496

Bryden, G., Xingming, C., Lin, D. N. C., Nelson, R. P., \& Papaloizou, J. C. B 1999, ApJ, 514, 344

Calvet, N., et al. 2005, ApJ, 630, 185

Chiang, E. I., \& Goldreich, P. 1997, ApJ, 490, 368

Chiang, E. I., \& Murray-Clay, R. A. 2007, Nat. Phys., 3, 604

Dullemond, C. P., \& Dominik, C. 2005, A\&A, 434, 971

Dullemond, C. P., Dominik, C., \& Natta, A. 2001, ApJ, 560, 957

Guilloteau, S., Dutrey, A., \& Simon, M. 1999, A\&A, 348, 570

Hartmann, L., Calvet, N., Gullbring, E., \& D’Alessio, P. 1998, ApJ, 495, 385

Hayashi, T. 1981, Prog. Theor. Phys. Suppl., 70, 35 
Herbig, G. H., \& Bell, K. R. (ed.) 1988, Lick Obs. Bull. 1111, Third Catalog of Emission-line Stars of the Orion Population (Santa Cruz, CA: Lick Observatory)

Hughes, A. M., Wilner, D. J., Qi, C., \& Hogerheijde, M. R. 2008, ApJ, 678, 1119

Hughes, A. M., et al. 2009, ApJ, 698, 131

Isella, A., Carpenter, J. M., \& Sargent, A. I. 2009, ApJ, 701, 260

Isella, A., Testi, L., Natta, A., Neri, R., Wilner, D., \& Qi, C. 2007, A\&A, 469, 213

Kenyon, S., \& Hartmann, L. 1995, ApJS, 101, 117

Koerner, D. W., \& Sargent, A. I. 1995, AJ, 109, 2138

Lin, D. N. C., \& Papaloizou, J. C. B. 1993, Protostars and Planets III, ed. E. H. Levy \& J. I. Lunine (Tucson, AZ: Univ. Arizona Press), 749

Lynden-Bell, D., \& Pringle, J. E. 1974, MNRAS, 168, 603

McGroarty, F., \& Ray, T. P. 2004, A\&A, 420, 975

Muzerolle, J., Calvet, N., \& Hartmann, L. 1998, ApJ, 492, 743

Natta, A., Testi, L., \& Muzerolle, J. 2004, A\&A, 424, 603

Piétu, V., Dutrey, A., \& Guilloteau, S. 2007, A\&A, 467, 163

Piétu, V., Dutrey, A., Guilloteau, S., Chapillon, E., \& Pety, J. 2006, A\&A, 460, 43

Piétu, V., Guilloteau, S., \& Dutrey, A. 2005, A\&A, 443, 945
Pollack, J. B., Hollenbach, D., Beckwith, S., Simonelli, D. P., Roush, T., \& Fong, W. 1994, ApJ, 421, 615

Pott, J.-U., Perrin, M. D., Furlan, E., Ghez, A. M., Herbst, T. M., \& Metchev, S. 2010, ApJ, 710, 265

Ricci, L., Testi, L., Natta, A., Neri, R., Cabrit, S., \& Herczeg, G. J. 2010, A\&A, 512, A 15

Robitaille, T. P., Whitney, B. A., Indebetouw, R., \& Wood, K. 2007, ApJS, 169 328

Schegerer, A. A., Wolf, S., Ratzka, Th., \& Leinert, Ch. 2008, A\&A, 478, 779

Shakura, N. I., \& Sunyaev, R. A. 1973, A\&A, 24, 337

Simon, M., Dutrey, A., \& Guilloteau, S. 2000, ApJ, 545, 1034

St-Onge, G., \& Bastien, P. 2008, ApJ, 674, 1032

Testi, L., Bacciotti, F., Sargent, A. I., Ray, T. P., \& Eislöffel, J. 2002, A\&A, 394, 31

Testi, L., Natta, A., Shepherd, D. S., \& Wilner, D. 2003, A\&A, 403, 323

Weingartner, J. C., \& Draine, B. T. 2001, ApJ, 563, 842

Wilner, D. J., Ho, P. T. P., Kastner, J. H., \& Rodríguez, L. F. 2000, ApJ, 534, 101

Wolf, S., Moro-Martin, A., \& D’Angelo, G. 2007, P\&SS, 55, 569

Zubko, V. G., Mennella, V., Colangeli, L., \& Bussoletti, E. 1996, MNRAS, 282, 1321 